\title{
Zebrafish reward mutants reveal novel transcripts mediating the behavioral effects of amphetamine
}

\author{
Katharine $\mathrm{J} \mathrm{Webb}^{* \dagger}$, William HJ Norton ${ }^{* \dagger}$, Dietrich Trümbach", \\ Annemarie H Meijer\$, Jovica Ninkovic ${ }^{* *}$, Stefanie Topp ${ }^{* \dagger}$, Daniel Heckף, \\ Carsten Marr"l, Wolfgang Wurst ${ }^{\ddagger}$, Fabian J Theis", Herman P Spaink ${ }^{\S}$ and \\ Laure Bally-Cuif ${ }^{*+}$
}

\begin{abstract}
Addresses: * Department Zebrafish Neurogenetics, Institute of Developmental Genetics, Helmholtz Zentrum Muenchen, German Research Center for Environmental Health, Ingolstaedter Landstrasse, Neuherberg, 85764 Germany. ${ }^{\dagger}$ Center for Integrated Protein Science (Munich), Institute of Developmental Genetics, Technical University - Munich, Ingolstaedter Landstrasse, Neuherberg, 85764 Germany. ${ }^{*}$ Institute of Developmental Genetics, Helmholtz Zentrum Muenchen, German Research Center for Environmental Health, Ingolstaedter Landstrasse, Neuherberg, 85764 Germany. §Institute of Biology, University of Leiden, Leiden, 2300 RA The Netherlands. "Institute for Bioinformatics and Systems Biology, Helmholtz Zentrum München, German Research Center for Environmental Health, Ingolstaedter Landstrasse, Neuherberg, 85764 Germany. ${ }^{¥}$ Current address: Institute of Stem Cell Research, Helmholtz Zentrum Muenchen, German Research Center for Environmental Health, Ingolstaedter Landstrasse, Neuherberg, 85764 Germany.
\end{abstract}

Correspondence: Katharine J Webb. Email: katharine.webb@helmholtz-muenchen.de. Laure Bally-Cuif. Email: bally@helmholtzmuenchen.de

Published: 31 July 2009

Genome Biology 2009, 10:R8I (doi:I0.1 I86/gb-2009-10-7-r8I)

The electronic version of this article is the complete one and can be found online at http://genomebiology.com/2009//0/7/R8I
Received: 12 May 2009

Revised: 16 June 2009

Accepted: 31 July 2009

(C) 2009 Webb et al.; licensee BioMed Central Ltd.

This is an open access article distributed under the terms of the Creative Commons Attribution License (http://creativecommons.org/licenses/by/2.0), which permits unrestricted use, distribution, and reproduction in any medium, provided the original work is properly cited.

\begin{abstract}
Background: Addiction is a pathological dysregulation of the brain's reward systems, determined by several complex genetic pathways. The conditioned place preference test provides an evaluation of the effects of drugs in animal models, allowing the investigation of substances at a biologically relevant level with respect to reward. Our lab has previously reported the development of a reliable conditioned place preference paradigm for zebrafish. Here, this test was used to isolate a dominant $\mathrm{N}$-ethyl-N-nitrosourea (ENU)-induced mutant, no addiction (naddne3256), which fails to respond to amphetamine, and which we used as an entry point towards identifying the behaviorally relevant transcriptional response to amphetamine.

Results: Through the combination of microarray experiments comparing the adult brain transcriptome of mutant and wild-type siblings under normal conditions, as well as their response to amphetamine, we identified genes that correlate with the mutants' altered conditioned place preference behavior. In addition to pathways classically involved in reward, this gene set shows a striking enrichment in transcription factor-encoding genes classically involved in brain development, which later appear to be reused within the adult brain. We selected a subset of them for validation by quantitative PCR and in situ hybridization, revealing that specific brain areas responding to the drug through these transcription factors include domains of ongoing adult neurogenesis. Finally, network construction revealed functional connections between several of these genes.
\end{abstract}

Conclusions: Together, our results identify a new network of coordinated gene regulation that influences or accompanies amphetamine-triggered conditioned place preference behavior and that may underlie the susceptibility to addiction. 


\section{Background}

Addiction, which can be broadly defined as a pathological state characterized by the compulsive seeking and usage of a drug in spite of adverse consequences, is a major societal problem. In the USA alone, more than 23 million Americans are concerned, with societal costs reaching 1.4 million dollars over the life of each addict [1]. Addictive drugs include a large number of substances (such as stimulants, alcohol and opiates) acting through different cellular mechanisms, but which all trigger a sequence of widespread, long-lasting consequences on brain physiology, most of which are only partially understood. The complexity of these plastic events makes it difficult to efficiently care for patients, and current treatments have little power to avoid relapse. As a consequence, a major goal of drug abuse research is to identify the key molecular mechanisms underlying the development of compulsive drug use, which may then be medically targeted for better treatments.

The mechanisms underlying drug addiction utilize a succession of physiological responses that begin with the activation of the brain's reward pathway - a feature common to all drugs of abuse. The reward system, largely based on dopamine signaling projecting to forebrain centers [2], signals a pleasurable experience, which then tends to be repeated. The transition from drug use to addiction [3] occurs gradually and involves both neuro- and synaptic plasticity. These long-lasting adaptive changes persist even after withdrawal of the drug, and they are likely to underlie the persistent tendency to relapse [4]. In addition, several other circuits - in particular the stress axis and the learning and memory circuitry - have been implicated in the reinforcement of drug use or addiction and in the cognitive processes underlying addiction [5]. One powerful approach to understand which molecular alterations contribute to the development and expression of the successive addiction-related behaviors has been the use of microarray expression profiling. Combined with the in silico assembly of regulatory networks, this high-throughput analysis can provide a comprehensive picture of the changes in gene expression that may underlie the different steps towards drug addiction. In the case of psychostimulant drugs, for example, microarray analyses have demonstrated the occurrence of important transcriptional changes that differ over time, clearly distinguishing acute from chronic drug use or withdrawal. In models as varied as human post-mortem brains from cocaine abusers and mice or rats of different genetic backgrounds, changes related to molecular pathways controlling neurotransmitter signaling (including a downregulation of the dopamine D2 receptor), signal transduction, ion-gated channel activity, cytoskeletal structures, extracellular matrix remodeling, synaptogenesis, axonal dynamics and cell metabolism [6-8] (reviewed in [9,10]) have been identified.

Because a major step in the development of addiction is the switch from drug use to drug abuse, we aimed to gain insight into the mechanisms triggering the initiation of addictive behavior. Towards this aim, we focused on commonalities in the effects of abused drugs, hence on their early effect on the reward pathway. Based on previous observations demonstrating that the response of the reward system increases with expectancy (thus, it is subject to auto-amplification) [11], we reasoned that a major susceptibility factor in the transition from drug use to abuse might be the intensity of the initial reward response. In order to narrow-down transcriptional approaches to this process, recent analyses compared the transcriptional effects of several drugs [12], or made use of mice carrying alterations in the function of genes postulated to be relevant to reward. For example, the transcriptional effects of cocaine have been compared in mice lacking the dopamine D1 receptor (necessary for the sensitization to cocaine) versus their wild-type siblings [7], in mice overexpressing the immediate early transcription factors CREB or $\Delta$ fosB (both of which are involved in mediating the acute effects of cocaine) [13] and in Cdk5 knock-out mice ( $\mathrm{Cdk}_{5}$ is a downstream target of $\Delta$ fosB) [14].

We aimed to provide an unbiased insight into this question, without a priori selection of a regulatory pathway, while remaining clearly associated with the reward behavioral output. To achieve these goals, we initiated a functional study of the reward pathway in zebrafish, a vertebrate model amenable to random mutagenesis and behavioral screening. Reward behavior is an ancestral behavior, conserved throughout vertebrate phyla, and the underlying neurotransmitter pathways are shared between species [15-18]. We chose to use the psychostimulant amphetamine as it directly stimulates the reward pathway (largely via altering the function of the dopamine transporter Dat [19], which elicits limited physical dependency, and on the behavioral assay known as conditioned place preference (CPP)). This test, in which association with the pleasurable effect of a drug modifies an animal's choice for a specific environmental cue, has been classically used as a read-out of the functionality of the reward system [20].

We recently developed a robust assay for amphetamineinduced CPP behavior in adult zebrafish, and demonstrated the role of acetylcholine signaling in the sensitivity to amphetamine-induced reward $[17,21]$. Here, relying on evidence suggesting genetic components in the susceptibility to addiction (reviewed in [22-24]), we used this assay in a $\mathrm{N}$ ethyl-N-nitrosourea (ENU) mutagenesis screen, successfully isolating an amphetamine-resistant mutant in the CPP test, no addiction (nad ${ }^{d n e} 3256$; hereafter referred to as nad). This mutation is dominant and nad heterozygotes fail to change their place preference upon repeated amphetamine administration. In zebrafish, amphetamine does not trigger a locomotor response [17], and lack of CPP is the only phenotype that we could associate with the nad mutation to date. We next used this mutant in a three-step expression profiling paradigm comparing the transcriptional response of wild-type 


\section{(a)}

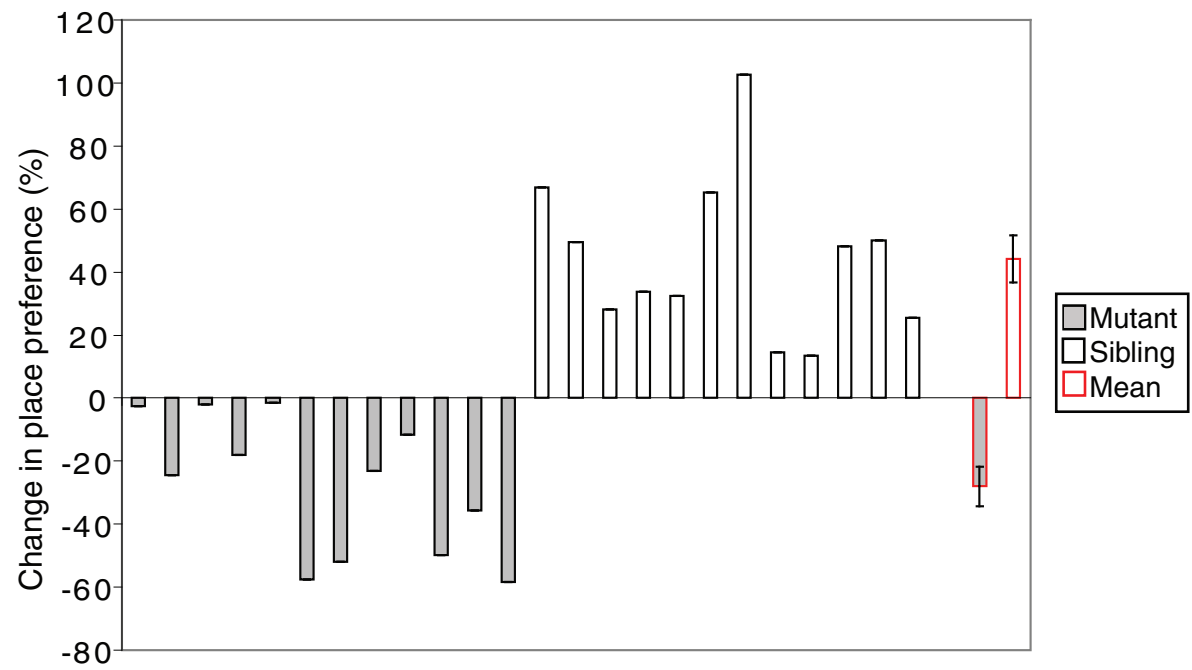

(b)

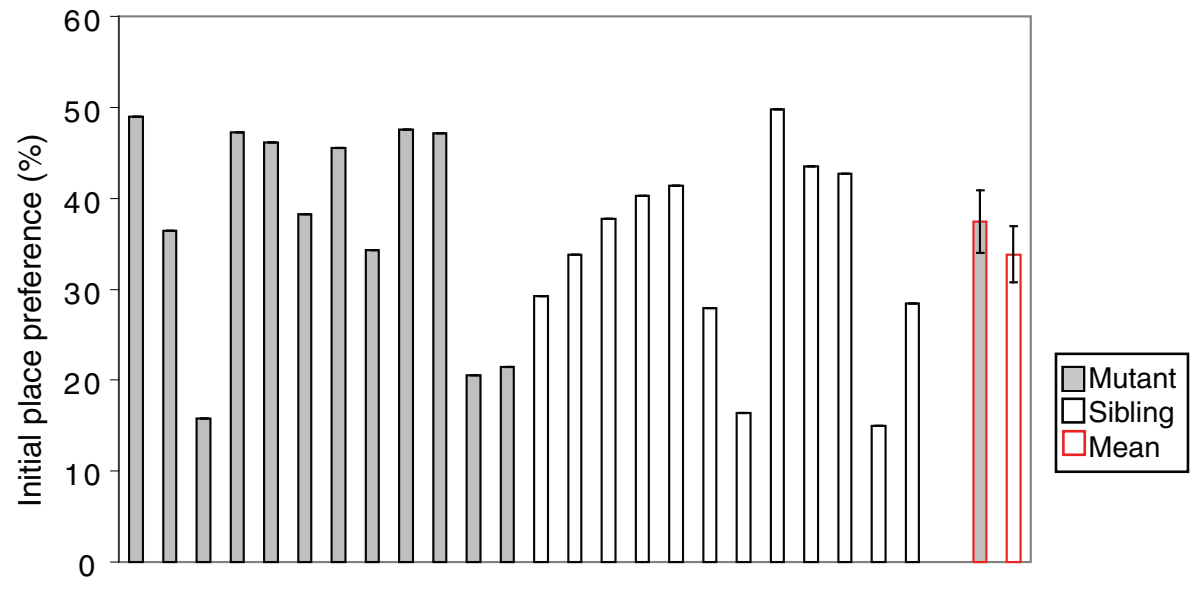

Figure I

The dominant mutant naddne 3256 shows no response to amphetamine, but a normal initial place preference. (a) Conditioned place preference (\%) of 24 individuals of a naddne3256 family (generation F3), showing 12 mutants and 12 siblings. Mutants were defined as showing no or a negative change in place preference. Siblings were defined as having a change in place preference of $5 \%$ or over. The last two bars represent the means for both groups. The difference between the means is statically significant ( $t$-test with unequal variances; $P=2.3 \mathrm{E}-07$ ). (b) Initial place preference (\%) for the same 24 individual fish. The last two bars represent the means for both groups. There is no significant difference between the two means (two sample unequal variance $t$-test $P=0.45$ ). Error bars represent the one fold of the standard error.

animals upon CPP-stimulating amphetamine administration with that of nad mutants receiving either drug or a saline control solution. We discovered a set of 139 genes that respond to amphetamine in wild-type animals, but respond inappropriately in nad mutants without being altered under basal conditions in either genotype. In addition to genes involved in pathways classically associated with reward, this gene set shows a striking enrichment in transcription factors that are specifically known for their involvement in brain development. We validated a subset of these genes using quantitative PCR (qPCR) and in situ hybridization, thereby revealing an association of these gene expressions with neurogenic zones of the adult brain, which is also apparent in the mouse. We developed a database linking zebrafish genes to information on orthologous gene interactions, which we then used to demonstrate that many of these genes contribute to a common regulatory network. Together, our results identify a pattern of coordinate gene regulation that may underlie or accompany the development of CPP behavior upon amphetamine administration and, hence, may contribute to generating a susceptibility background towards the development of addiction. 
(a)

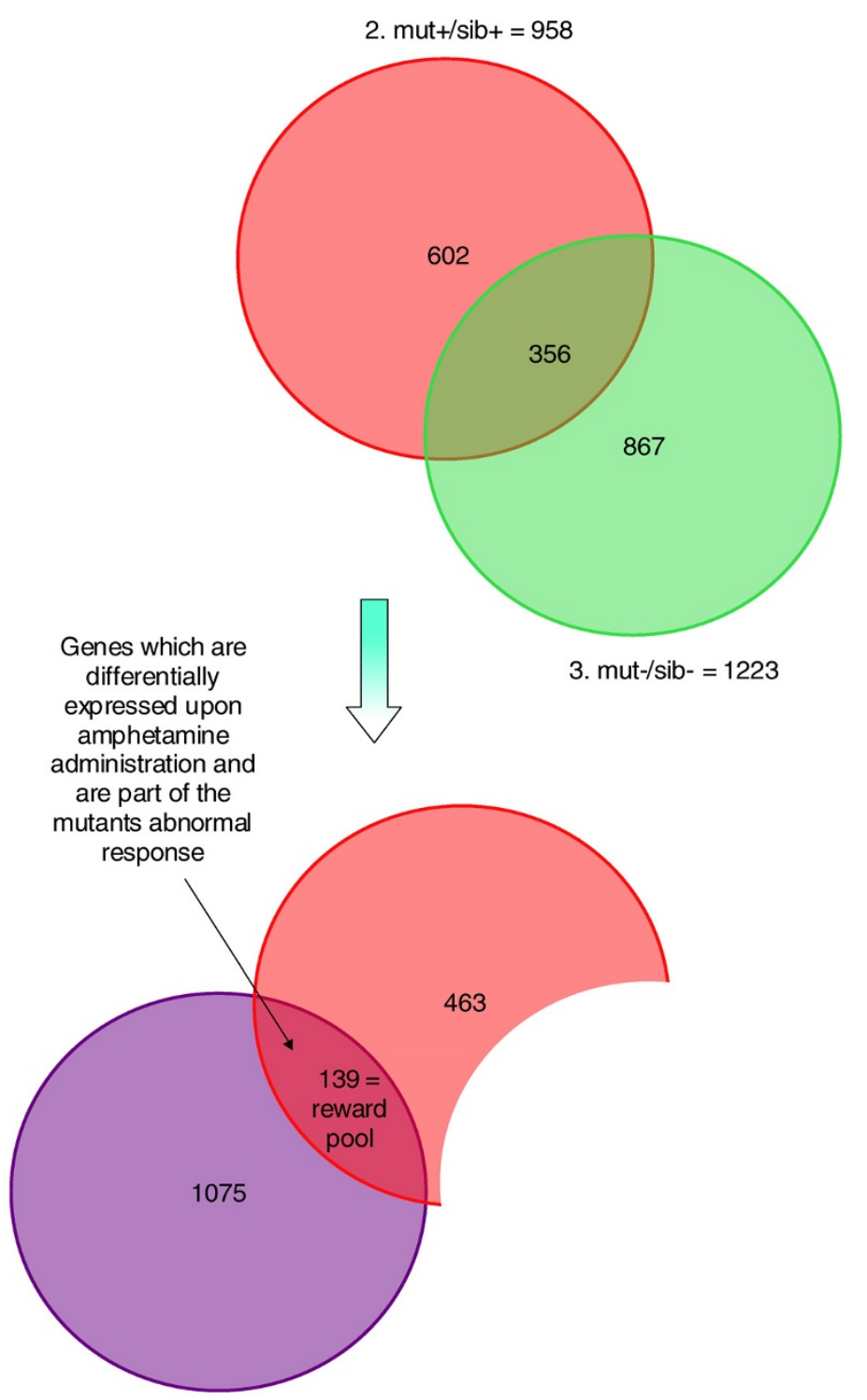

1. wt+/wt- $=1214$

(b)

\begin{tabular}{|c|c|c|c|}
\hline $\begin{array}{c}\text { Direction of } \\
\text { differential } \\
\text { expression in } \\
\text { wt+ (vs. wt -) }\end{array}$ & $\begin{array}{c}\text { Direction of } \\
\text { differential } \\
\text { expression in } \\
\text { mut+ (vs. sib+) }\end{array}$ & Conclusion & $\begin{array}{c}\text { No. of } \\
\text { transcripts } \\
(\%)\end{array}$ \\
\hline$\uparrow$ & $\uparrow$ & $\begin{array}{c}\text { Increased up-regulation } \\
\text { in mutant }\end{array}$ & $24(17)$ \\
\hline$\downarrow$ & $\uparrow$ & $\begin{array}{c}\text { Increased down- } \\
\text { regulation in mutant }\end{array}$ & $33(24)$ \\
\hline$\downarrow$ & $\downarrow$ & $\begin{array}{c}\text { Mutant fails to } \\
\text { downregulate }\end{array}$ & $33(24)$ \\
\hline$\uparrow$ & & $\begin{array}{c}\text { Mutant fails to } \\
\text { upregulate }\end{array}$ & $49(35)$ \\
\hline & & &
\end{tabular}

Figure 2 (see legend on next page) 
Figure 2 (see previous page)

'Reward pool' genes characterize the transcriptional response to amphetamine-triggered CPP. (a) Diagram of differentially expressed genes from microarray experiments. Individual microarray experiments were combined to reveal a reward pool. A comparison of the differential expression from two experiments showed no bias in the direction of expression. Pool I shows the genes differentially expressed in 'wild type with amphetamine versus wild-type without amphetamine'. Pool 2 represents genes differentially expressed in 'mutant with amphetamine versus sibling with amphetamine'. Pool 3 represents genes differentially expressed in 'mutant without amphetamine versus non-mutant siblings without amphetamine'. The genes in pool 3 were subtracted from pool 2 in order to eliminate basal differences between mutants and siblings, not due to amphetamine administration. The intersection of the remaining genes in pool 2 and the genes in pool I forms the 'reward pool'. The genes in this pool are differentially expressed in both experiments - that is, they are involved in the wild-type response to amphetamine, as well as the non-response to amphetamine in the mutant. (b) Comparison of the direction of regulation (up or down) of transcripts from the reward pool for the experiments wt+/wt- and mut $+/ s i b+$. No bias towards a particular pattern can be observed.

\section{Results}

The mutant nad fails to respond to amphetamineinduced reward

To recover mutants of the amphetamine response, we designed an ENU mutagenesis screen making use of the amphetamine-based CPP test for adult zebrafish [21]. Briefly, in this test, the psychostimulant amphetamine is associated with the initially non-preferred side of a two-color tank. The repeated administration of amphetamine causes a switch in the place preference of the fish: even in the absence of drug, the animal will now prefer the amphetamine-paired side of the tank. As previously demonstrated using adults heterozygous for the achesb55 mutation, this test is robust enough to detect dominant mutations affecting amphetamine-triggered preference [17]. To recover new dominant mutations of this type, we screened F1 animals generated from ENU-treated Fo males for their place preference response to amphetamine. Potential mutants were then out-crossed to wild-type fish and their F2 progeny was retested at adulthood. From $396 \mathrm{~F} 1$ animals tested (corresponding to 396 genomes), 4 animals failed to change their place preference upon amphetamine administration while showing normal initial place preference without drug (not shown). One of these potential mutants transmitted this phenotype to $50 \%$ of its progeny, following the expected Mendelian distribution for dominant genetic traits (Figure 1a). To date, this transmission has been stable over more than five generations and is detectable equally well in both the $\mathrm{AB}$ and the polymorphic $\mathrm{AB} / \mathrm{Tü} \mathrm{background} \mathrm{[21],} \mathrm{arguing} \mathrm{for} \mathrm{a}$ bona fide dominant mutation. Importantly, the initial place preference in mutants does not differ from that of their siblings (Figure 1b), demonstrating their normal response to the visual cues of the test tank. Following drug treatment, amphetamine brain content is also similar in mutant fish and their siblings (not shown). We named this mutation no addiction (naddne3256).

\section{A distinct gene expression signature underlies the abnormal behavioral response of nad mutants to amphetamine}

Previous experiments based on candidate gene or microarray analysis demonstrate that amphetamine treatment has an impact on gene expression (for a review, see [10]). These gene expression changes are likely to mediate or reflect a large part of amphetamine's actions on multiple biological processes, one of which is to activate the reward pathway. The design of our mutant screen further implies that the effect of amphetamine on CPP development is impaired in nad (see Discussion for the possible behavioral meanings of nad). Thus, the changed transcriptional response of nad to the drug can help identify the genes meaningful to the response to amphetamine.

We designed three microarray comparisons to specifically isolate such genes (Figure 2a; see Additional data files 1 to 3 for complete gene lists). In a first comparison, we found 1,214 genes to be differentially expressed between wild-type fish that received amphetamine treatment triggering CPP versus fish that received a control, saline treatment (experimental conditions identical to those described above; microarray experiment 1, 'wt+/wt-'; Figure 2a, purple group). To extract genes meaningful to CPP development from this pool, we next identified the transcripts that were differentially affected by amphetamine in nad versus their wild-type siblings. Analysis of the microarray data for this second comparison showed that 958 genes were differentially expressed between mutants and wild-type siblings upon amphetamine treatment (experiment 2, 'mut+/sib+'; Figure 2a, pink group). However, as these are likely to include basal transcriptional differences between mutants and wild-type fish (that is, transcriptional differences that are not triggered by amphetamine administration), we performed a third microarray comparison between mutants and their siblings without amphetamine (experiment 3, 'mut-/sib-'; Figure 2a, green group). We found 1,223 genes to be differentially expressed under these conditions, which we then took to represent the basal differences between the mutants and their siblings. Of these, 356 were also differentially expressed in the experiment 'mut+/sib+' and were then subtracted from this group to recover genes characterizing the different response of mutants versus siblings to amphetamine. This subtraction resulted in the pool 'mut+/sib+ minus mut-/sib-'. The intersection of the pools 'mut+/sib+ minus mut-/sib-' and 'wt+/wt-' was taken to form the 'reward pool' - that is, genes that both characterize the wild-type response to amphetamine and that display an altered response (that is, they respond less or more than in wild type) in the mutant with amphetamine, correlating with the failure of CPP in this genotype. This pool comprises 139 
(a)

\begin{tabular}{|c|c|c|c|c|c|}
\hline GO term & $\begin{array}{l}\text { \# of } \\
\text { entities }\end{array}$ & $\begin{array}{l}\text { Common } \\
\text { objects }\end{array}$ & P-value & $\begin{array}{l}\text { Adjusted P- } \\
\text { value } \\
\text { (Bonferroni) }\end{array}$ & Protein name \\
\hline $\begin{array}{l}\text { Regulation of } \\
\text { transcription, } \\
\text { DNA-dependent }\end{array}$ & 2763 & 11 & $3.44 \mathrm{E}-0.6$ & 0.001 & $\begin{array}{l}\text { Her15, Ahr1a, Sox9a, Dlx4a, } \\
\text { LOC555375 (similar to Nkx3- } \\
\text { 1), Gfi1b, Dlx1a, Hoxd12a, } \\
\text { Foxg1, Emx1, Lhx8 }\end{array}$ \\
\hline Development & 913 & 7 & 3.62E-06 & 0.001 & $\begin{array}{l}\text { Her15, Dlx4a, LOC555375 } \\
\text { (similar to Nkx3-1), Dlx1a, } \\
\text { Hoxd12a, Foxg1, Emx1 }\end{array}$ \\
\hline Transport & 1860 & 9 & $6.02 E-06$ & 0.002 & $\begin{array}{l}\text { LOC558674 (similar to Kcns1) } \\
\text { Crat, Cx44.1, Slc6a5, Irbp, } \\
\text { Chrne, Fabp10, Atp1a1a.3, } \\
\text { Atp1a1a.4 }\end{array}$ \\
\hline $\begin{array}{l}\text { Visual } \\
\text { perception }\end{array}$ & 236 & 4 & 2.34E-05 & 0.007 & Cx44.1, Epcam, Irbp, Gnat2 \\
\hline $\begin{array}{l}\text { Response to } \\
\text { xenobiotic } \\
\text { stimulus }\end{array}$ & 11 & 2 & $2.56 \mathrm{E}-05$ & 0.007 & Ahr1a, Cyp1a \\
\hline Metabolism & 673 & 5 & 0.0001095 & 0.032 & $\begin{array}{l}\text { Slc6a5, Similar to Oxct1b, } \\
\text { Atp1a1a.3, Atp1a1a.4 } \\
\text { zqc:101565 }\end{array}$ \\
\hline $\begin{array}{l}\text { Iron ion } \\
\text { homeostasis }\end{array}$ & 151 & 3 & 0.00016468 & 0.048 & Tfr1b, Сyp1a, zgc:55856 \\
\hline
\end{tabular}

(b)

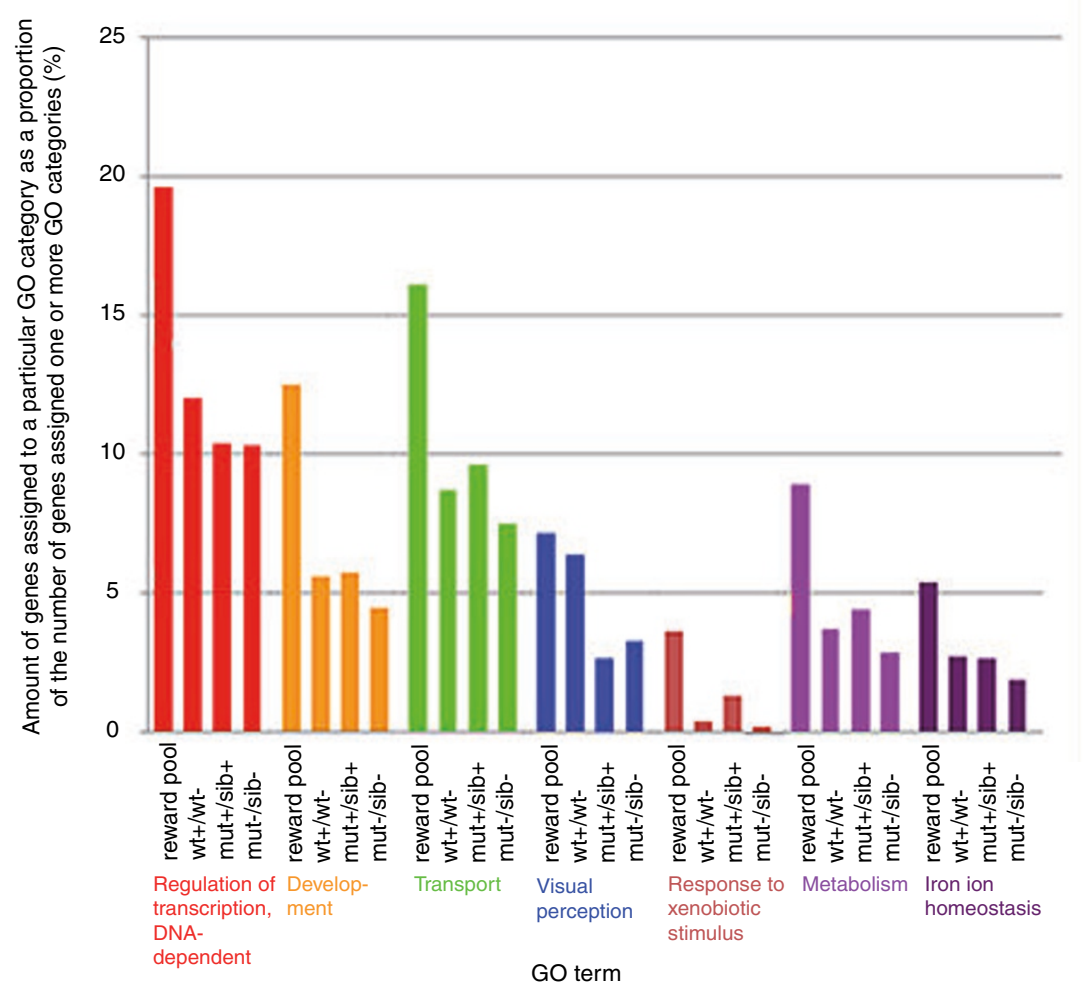

Figure 3

Categorization of the 139 genes contained in the reward pool. (a) Gene Ontology (GO) term enrichment analysis for terms included in the category 'biological process'. Terms were considered to be significantly enriched if the adjusted $P$-value was $<0.05$. Redundant terms have been excluded. (b) Bar graph comparing the amount of genes assigned to a particular category as a percentage of all genes that could be assigned one or more GO terms. The depicted categories were chosen from the analysis in (a). For the experiment wt+/wt- 517 genes were assigned a GO term, for mut $+/$ sib +386 , for mut-/sib- 493 and for the reward pool 56. 
genes, which are listed and functionally annotated in Additional data file 4 .

Of the 139 genes in the reward pool, $17 \%$ were upregulated in both 'mut+/sib+' and 'wt+/wt-' (Figure 2b). Transcription of these genes is increased in wild-type fish upon amphetamine treatment and excessively increased in the mutants. Conversely, 24\% of the 139 genes were down-regulated in both experiments; hence, their transcription is normally downregulated upon amphetamine treatment, and is excessively down-regulated in the mutants. Finally, a majority of the genes ( $59 \%$ of 139 ) responded to amphetamine in an opposite manner between wild-type and mutant fish (24\% of the 139 genes were up-regulated in mut+ compared to sib+, but downregulated in wt+ compared to wt-, and 35\% were downregulated in mut+ compared to sib+ and up-regulated in wt+ compared to wt-). These genes failed to be down- or up-regulated, respectively, in the mutants upon amphetamine treatment.

\section{The reward pool is significantly enriched in transcription factor-encoding genes}

In order to further investigate the mechanisms involved in reward, Gene Ontology (GO) enrichment analyses categorizing the genes in the organizing principle 'biological process' were performed on each of the individual experiments and the reward pool (Figure 3; Additional data file 5). We found that the reward pool contains a high proportion of genes encoding functions previously related to reward or the transition to addiction, such as neurotransmitter signaling pathways, ion channels and regulators of neuronal and synaptic plasticity (see Additional data file 4 and Discussion). In order to characterize processes specific to the rewarding effects of amphetamine, we also searched for particular enrichments in the reward pool over the other three gene sets. Enriched categories in the reward pool (Figure $3 \mathrm{a}$ ) and individual experiments (Additional data file 5) are compared in Figure $3 \mathrm{~b}$. The most striking result was that the term 'transcription' was enriched across all groups, but displayed a further relative increase in the reward pool. This was also the case for the term 'development', and, in fact, both superordinate categories largely overlapped in their gene content (see Figure 3 a for a list of proteins encoded by these genes). Thus, the involvement of transcription factors previously recognized for their relevance in developmental processes distinguishes the reward response to amphetamine (and its failure in nad) over other transcriptional effects of amphetamine treatment.

\section{Amphetamine-responding genes can be validated by quantitative PCR and classified as acute and/or chronic responders}

The proteins encoded by individual genes annotated in the zebrafish genome (Ensembl release zv7 [25] and ZFIN [26]) are listed in Figure 3 a corresponding to each term for the reward pool (see Additional data file 5 for the GO term enrichment in the individual array experiments). We chose ten genes for validation based on GO enrichment analysis and literature searches: ahr1a, dlx1a, emx1, foxg1, gfi1b, her15, lhx8, slc6a5, sox9a and tbr1. As developmental transcription factors have not been recognized as a signature of the behavioral response to amphetamine in previous studies, our selection was largely biased towards this category: nine of the chosen genes encode transcription factors (ahr1a, dlxia, emx1, foxg1, gfi1b, her15, lhx8 (previously lhx7), soxga and tbr1), four of which have been assigned the GO term 'development' (her15, foxg1, emx1 and dlx1a). In addition to their generally prominent role during brain development, strong arguments to choose these genes were: the maintenance of expression of their orthologues in the adult mammalian brain (respectively in mouse, Ahr, Dlx1, Emx1, Foxg1, Gfi1, Hes5, Lhx8, Soxy and Tbr1) [27-35]), suggesting an extended role in controlling brain physiology; and their comparable expression patterns in both mouse [32,36-42] and zebrafish [43-48] and our unpublished data), at least during brain development, arguing for conserved functions in these species. In addition, we chose to test slc6a5 as a representative of the neurotransmitter pathway genes recovered in the reward pool. slc6a5 encodes the glycine neurotransmitter transporter GlyT2, which is involved in the reuptake of glycine at the synapse.

In a first step, expression of these genes in the wild-type adult brain was confirmed using in situ hybridization. All ten transcripts gave strong signals in the brain, including the telencephalon (Additional data file 6). Specifically, the expression of $g$ fir $b$ and her15 is restricted to the ventricular areas of the telencephalon (Additional data file 6e, f), diencephalon, and midbrain (not shown). dlx1a, emx1, foxg1, lhx8, slc6a5, sox9a and $t b r 1$ are expressed in restricted areas of the brain, including subdomains of the pallium and/or subpallium in the telencephalon (Additional data file $6 b-d, g-j$ ). Overall, the regional expression of these genes is in keeping with their known expression in the adult mammalian brain (see Discussion). ahr1a is expressed ubiquitously throughout the brain (Additional data file 6a; and data not shown).

Next, qPCR was used to validate the differential expression of seven of these ten genes upon amphetamine administration (emx1, foxg1, gfi1b, her15, lhx8, slc6a5 and sox9a). Five of these genes (emx1, foxg1, her15, slc6a 5 and sox9a) were first re-tested on the original RNA used for the microarrays. All were differentially regulated in the same direction as in the microarray for both wt+/wt- and mut+/sib+, thus validating our microarray experiments (Figure 4a, b; Additional data file 7). We next tested all genes using new RNA samples. Our experimental design for the arrays involved four injections of amphetamine alternating with three doses of saline solution, with the last amphetamine injection given 30 minutes before death. We hypothesized that this would allow us to identify genes reacting to any or just acute or chronic amphetamine administration. As previous studies showed differences in the reaction of transcriptional levels to these different treatments 
(a)

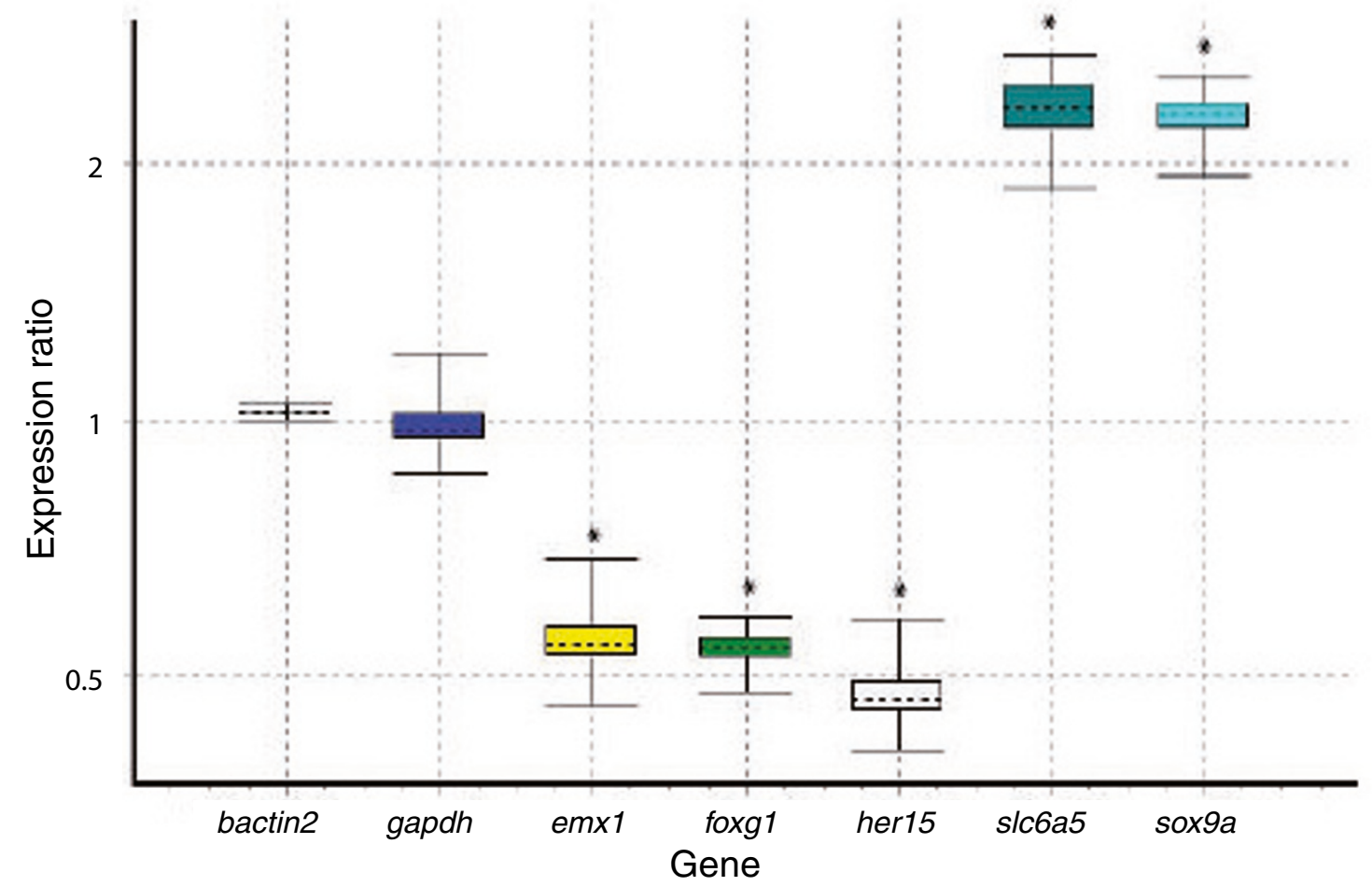

(b)

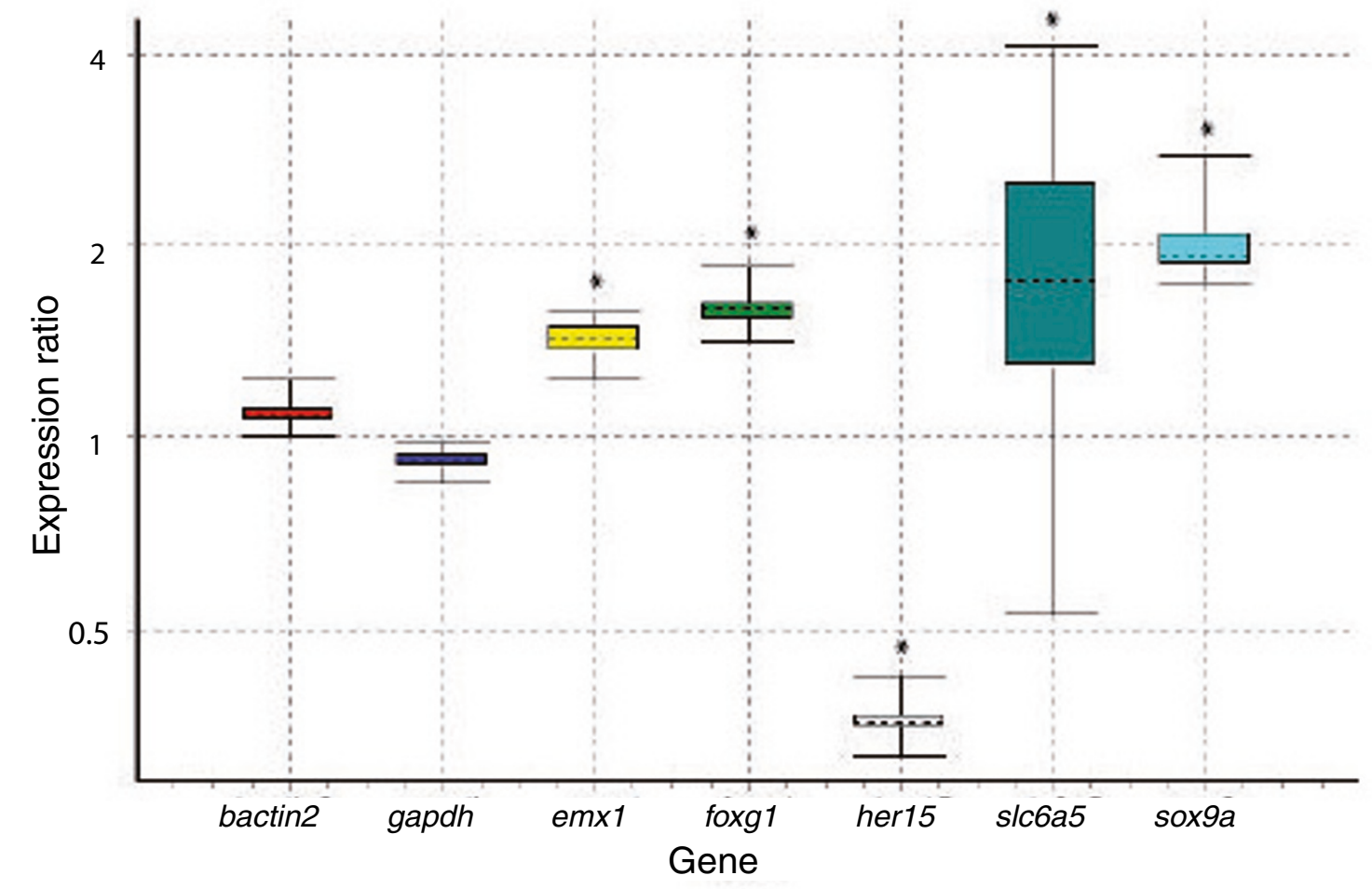

Figure 4

Validation using quantitative PCR. Individual genes with different biological roles were selected from the reward pool (Figure 3a) for qPCR using original RNA from (a) $\mathrm{wt}^{+} / \mathrm{wt}$ - and (b) mut $+/ \mathrm{sib}+$. The qPCR experiment revealed selected genes showed expression changes similar to those seen in the microarray results. The figures show box plots of relative gene expression, where the top and the bottom of each box indicate the 75th and 25th percentiles, respectively, whereas the dotted line represents the median. Asterisks indicate that the probability of the alternative hypothesis (that the difference between sample and control groups is only due to chance $(P(H I))$ being correct is $\leq 0.05$ (see also Materials and methods). 
[49], we conducted qPCR experiments on the 7 aboveselected genes using brains from fish that had been injected once (acute) or 18 times once daily (chronic) with amphetamine, with the last administration 30 minutes before death. The results are depicted in Figure $5 \mathrm{a}$, b (see also Additional data file 7). emx1 and $g$ fir $b$ appeared differentially expressed upon acute amphetamine administration, while there was no difference in foxg1, her15, lhx8, soxga and slc6a5 expression between acute-treated and untreated samples. However, all seven genes were differentially expressed upon chronic amphetamine administration, always in the same direction as in the arrays. These results further validate our arrays and, in addition, suggest that the amphetamine administration procedure used to trigger a CPP response in this work is closer to a chronic than to an acute paradigm.

\section{A subset of the reward pool genes is visibly modulated in situ by amphetamine}

As demonstrated above, qPCR using total RNA extracted from whole brains was used to validate and extend the results of our microarrays. However, this approach does not provide information as to which regions of the brain are transcriptionally affected by the drug. In situ hybridization was next performed on brain sections of fish chronically injected with amphetamine or saline solution (once a day for 18 days). Of the ten genes selected above, the expression of foxg1, gfi1 $b$, her15 and $\operatorname{lh} x 8$ were visibly changed upon amphetamine administration. The expression of gfi1b and her15, which characterize the ventricular zone in all brain subdivisions, was completely and consistently down-regulated throughout the brain (Figure 6a-d; Additional data file 8a-d). The expression of foxg 1 and $\operatorname{lh} x 8$ were affected in a region-dependent manner. foxg 1 transcription was reduced in the ventrolateral thalamic nucleus, and eliminated at the midline in the ventral zone of the periventricular hypothalamus and the parvocellular preoptic nucleus - no expression changes were detected throughout the remainder of the brain (Figure 6e, f; Additional data file $8 \mathrm{e}, \mathrm{f}$ ). The expression of $l h x 8$ was also much reduced in this latter domain upon amphetamine treatment, but was unchanged elsewhere in the brain (Figure $6 \mathrm{~g}, \mathrm{~h}$; Additional data file $8 \mathrm{~g}, \mathrm{~h}$ ). The reduction or increase of expression of the other six selected genes, indicated by the array and qPCR data, was not visible using in situ hybridization (not shown). As in situ hybridization is not a quantitative technique, it is possible that changes in expression must be large before they can be observed using this method. Together, these results highlight that ventricular domains of the adult brain are major areas responding to an amphetamine administration paradigm activating the reward pathway, and identify $g$ fir $b$, her15, $\operatorname{lh} x 8$ and foxg1 as prominent transcriptional targets in these domains.

We finally aimed to determine whether genes of the reward pool could be functionally connected. We developed a database (ZFISHDB) linking zebrafish genes to functional annotations and relationships via the STRING database. From the
139 genes of the reward pool, 53 could be attributed to a cluster of orthologous genes; 25 interactions were found between 18 of these genes (Figure 7). In particular, eight of the transcription factors, of which five have demonstrated roles in brain development (Dlx1a, Emx1, Lhx8, Sox9a and Tbr1), could be functionally connected, suggesting that amphetamine may re-use a developmental network in mediating reward in the brain.

\section{Discussion}

A major unsolved problem in the field of drug addiction is the characterization of the transcriptional changes underlying the switch from drug use to drug abuse. In this study we used an unbiased paradigm to identify a subset of genes involved in reward activation and its behavioral output. Our approach does not rely on the prior selection of a particular pathway, but rather on a mutant (nad) whose only phenotype is the lack of CPP behavioral response to amphetamine. Although we have not yet identified the mutation underlying this phenotype, we were able to extract a subset of 139 genes from the general transcriptional response to amphetamine that respond abnormally to amphetamine in the mutant, correlating with a failure to develop CPP after amphetamine treatment. transcriptional regulation of these genes is, therefore, associated with reward-triggered CPP behavior. We validated the microarray using both qPCR and in situ hybridization, thereby identifying neurogenic areas as potentially significant for the response to amphetamine. Our analysis highlighted the predominance of transcription factors in the response to amphetamine. These genes have been recognized for their function during brain development in both zebrafish and mouse, and are also expressed in the adult brain, pointing to the re-use of a developmental network as a potentially important component of reward behavior.

\section{Behavioral significance of the reward pool}

Based on a subset of genes recovered in our array, we used qPCR to show that our experimental conditions mimic chronic amphetamine administration. These genes therefore represent early but not acute transcriptional changes induced by the drug. We do not know, however, whether these changes are long-lasting. Our experimental design also allowed us to focus on a biologically relevant dose of amphetamine with regard to activation of the reward pathway. Finally, the non-response of nad mutants suggests that the expression changes recovered are, in part, linked to CPP behavior, although their functional significance with regard to the development of CPP was not addressed in this study. Several parameters underlie CPP behavior and might be altered in nad, such as the functionality of the reward pathway itself and the associative learning process involved in CPP, but also changes in tolerance or sensitization to rewarding or motivational events. We have not noticed any other behavioral or morphological alterations in nad, and also failed to observe differences in gross neuroanatomy and the 
(a)

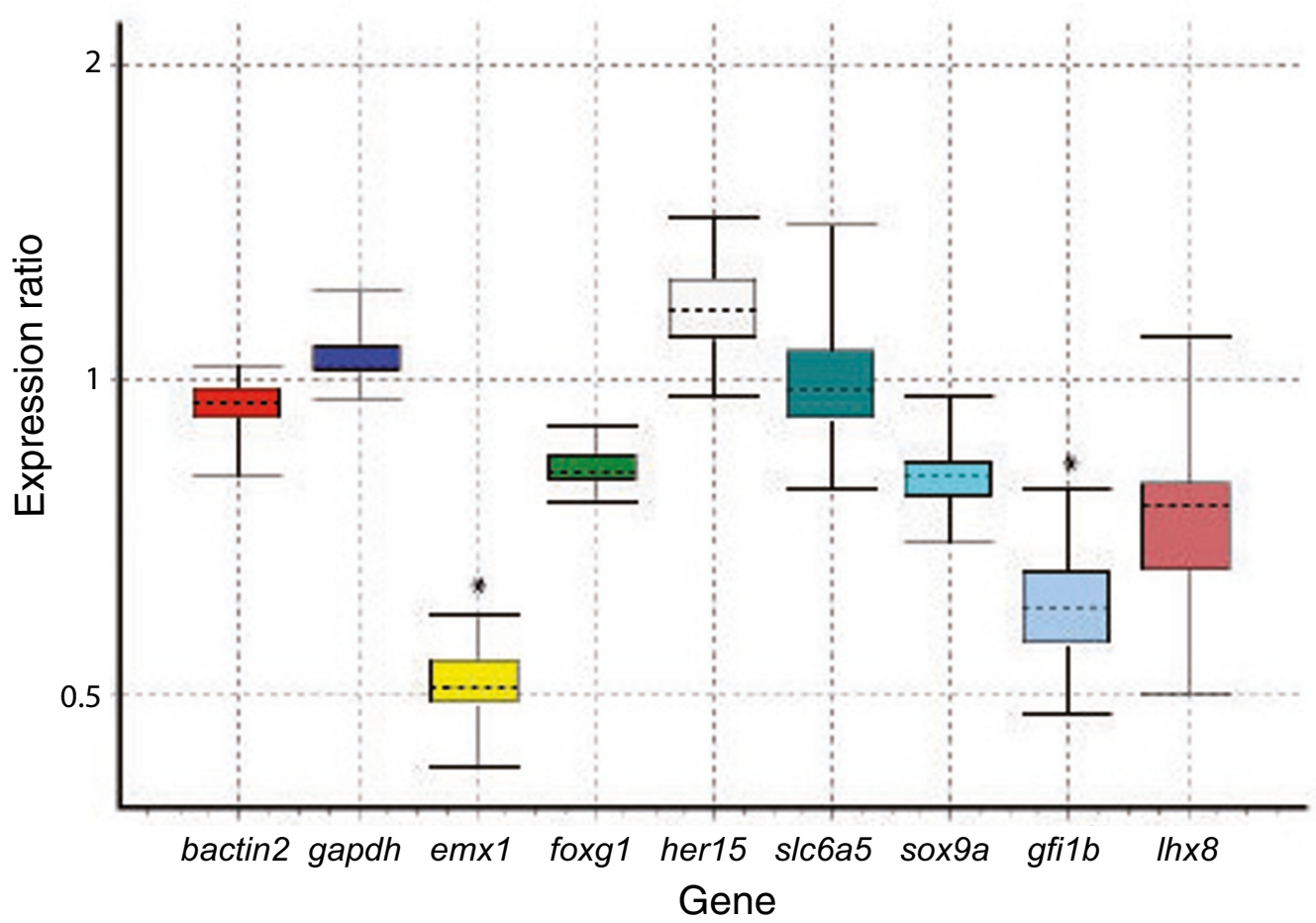

(b)

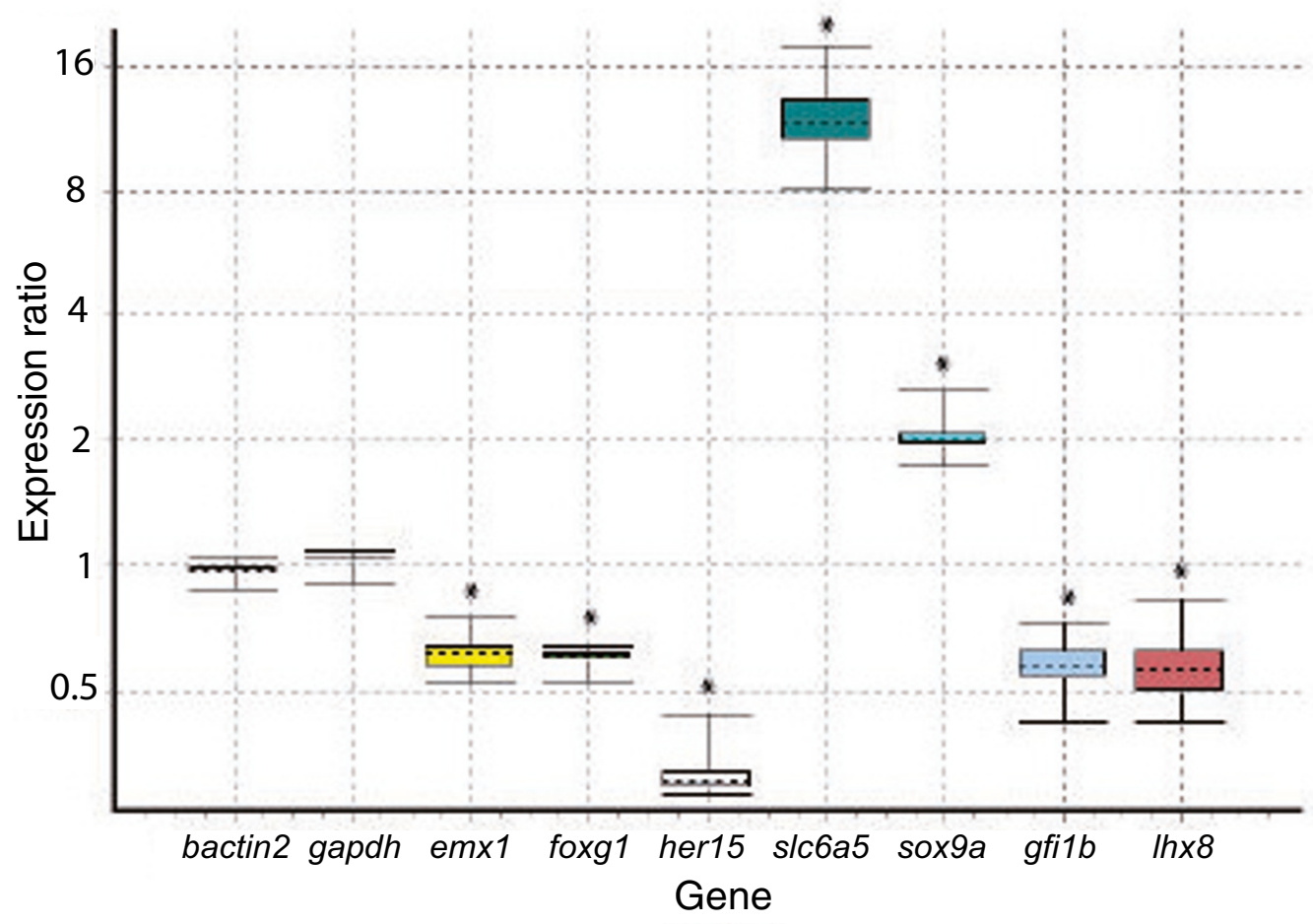

Figure 5

Validation and categorization of transcripts in acute and/or chronic response to amphetamine using quantitative PCR. qPCR was performed on the brain of fish injected with one dose (acute) or 18 doses of amphetamine (chronic). (a) Two genes, gfilb and emxl, were downregulated after one dose of amphetamine. (b) The remaining transcripts were down- or up-regulated in the same direction as the microarray in the chronic situation. The figures show box plots of relative gene expression, where the top and the bottom of each box indicate the 75th and 25th percentiles, respectively, whereas the dotted line represents the median. Asterisks indicate that the probability of the alternative hypothesis (that the difference between sample and control groups is only due to chance $(P(H I))$ being correct is $\leq 0.05$ (see also Materials and methods). 
organization of several neurotransmitter systems in this mutant (including dopamine and serotonin, revealed by tyrosine hydroxylase and 5-hydroxytryptamine immunocytochemistry, respectively; data not shown). Nevertheless, nad animals may exhibit yet other deficient responses to amphetamine that might become apparent if we test later stages of the addiction process, such as the maintenance of drug use, withdrawal or relapse. Whether nad mutants are also resistant to other addictive drugs that primarily act through different molecular cascades than psychostimulants, such as opiates [50], and whether the genes of the reward pool are correlatively also transcriptionally modified upon administration of these drugs, remain further very important questions. It will be essential to assess these points in the future to better connect the genes of the reward pool to behavioral function.

\section{Identification of amphetamine-induced transcriptional changes with no indication of toxic effects}

Importantly, we did not find any evidence of genes linked to cell-stress or cell-death, either in individual experiments or in the reward pool. This is in accordance with other microarray expression profiling studies, such as [49], which found little evidence of such genes upon chronic drug treatment. In contrast, many such genes were recovered upon acute administration of psychostimulants (and other drugs like morphine [51]), which may be due to the direct neurotoxic effects of amphetamine or cocaine. Likewise, immediate early transcription factors such as Erg2, Krox24, c-fos, c-jun and CREB, which are often transiently up-regulated following administration of drugs of abuse (reviewed in [9]), were enriched in neither individual experiments nor the reward pool. This confirms that our gene sets reflect transcriptional changes resulting from chronic rather than acute amphetamine action and may mediate the link to the different aspects of addiction. The category of genes related to the biological function 'response to stimulus' was enriched upon drug administration over saline in both wild-type and mutants. However, these genes were filtered out in the reward pool, confirming that they reflect a pharmacological response to the administration of chemical compounds that is unlikely to be altered in nad and so might not be involved per se in the development of behavioral alterations upon drug taking.

We decided to extract RNA from whole brains, rather than choosing specific anatomical regions. This approach was based on several considerations. Firstly, in addition to acting on the dopaminergic and serotonergic systems, amphetamine raises extracellular levels of glutamate [52] and noradrenaline [53] and these circuits are widely distributed throughout the brain. Secondly, the use of zebrafish makes it difficult to precisely predict where relevant expression changes are to be expected. Although the neurochemical aspects of reward behavior, including CPP, are evolutionarily conserved [18,50,54-56], some of the main neurotransmitter pathways involved in these behaviors show divergent spatial organiza- tion between species. For example, the dopaminergic neurons projecting to the zebrafish subpallium (which is hypothesized to be an equivalent of the mammalian basal ganglia, including the nucleus accumbens) are located in the diencephalic posterior tuberculum, unlike in mammals where these neurons lie in the ventral tegmental area of the midbrain [57]. Likewise, the zebrafish brain, as in many other vertebrate classes [58-60], harbors widespread serotonergic clusters as apposed to the single mammalian raphe nucleus [61]. However, as discussed below, we complemented our microarray experiments with in situ hybridization in order to allow us to investigate spatial changes in the expression of recovered transcripts and to identify relevant brain areas responding to amphetamine.

\section{Transcriptionally regulated pathways and reward behavior}

Most microarray analyses of reward and addiction to psychostimulants have been conducted in rodents. In addition, one transcriptome analysis of the adult zebrafish brain was recently published, comparing the effects of ethanol and nicotine during withdrawal [54]. It is not possible to analyze all these results side by side given the variety of drugs and drug administration protocols used and in the length of time allowed following drug exposure. Nevertheless, a general outcome was the response to chronic drug use of molecular pathways controlling neurotransmitter signaling (including receptors, transporters and signal transduction components), ion channels and regulators of neuronal activity and plasticity events such as synaptic function or extracellular matrix remodeling [9,13,62-65]. Our manual annotation of the 139 reward pool genes allowed us to identify related mammalian genes in most cases (84 of 139), and to postulate a function based on gene homology or on predicted protein structure for an additional 8 genes (92 of 139), so that our data can be directly compared to previous work. Of the functionally annotated genes of the reward pool, 28 belong to the categories above and 14 have already been linked to reward or addiction (Additional data file 4).

Affected genes related to neurotransmission include those encoding the epsilon subunit of the nicotinic acetylcholine receptor (chnre) and glycine transporter 2 (slc6a5, formerly glyT2), and LOC793458, which encodes peptide YYb (PYYb) [66]. All three pathways have been directly or indirectly implicated in reward [67]. We found chnre expression to be increased upon amphetamine administration in the wild-type and excessively increased in nad animals. Therefore, amphetamine may confer enhanced excitability properties on acetylcholine target neurons via a novel composition of the acetylcholine receptor, which could be linked to the development of the CPP response. Glycine is a major modulator of NMDA receptor-mediated signaling and glutamate neurotransmission is a determining factor in psychostimulant (and other) addictions (for reviews, see [68-70]). It has also been implicated in the regulation of neuronal differentiation, neural network plasticity and synapse dynamics. We found that 


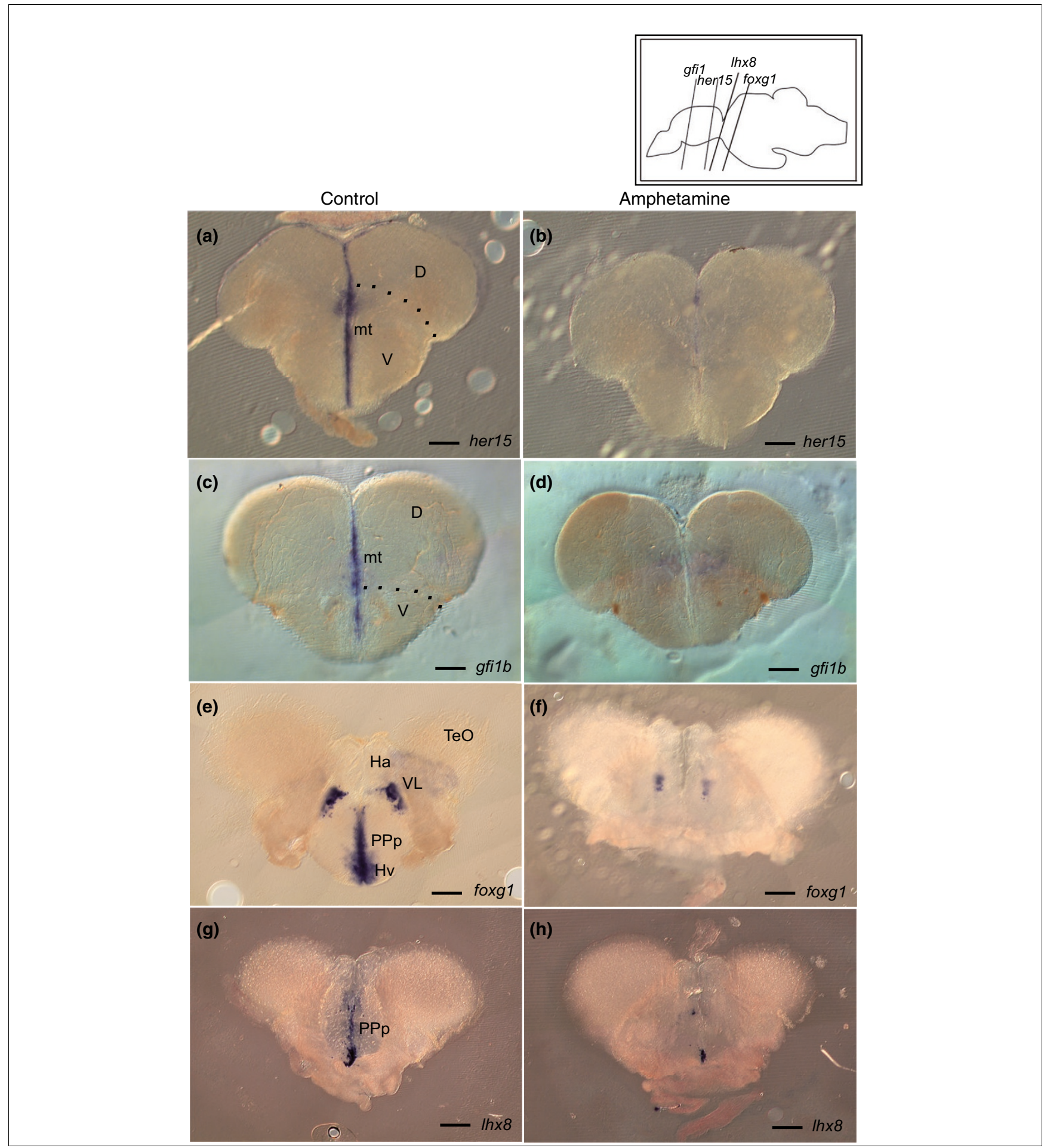

\section{Figure 6}

Candidate genes validated using in situ hybridization. (a, c) gfilb and her $/ 5$ are expressed in ventricular zones throughout the brain, including in the midline of the telencephalon. (b, d) Upon chronic amphetamine administration, this expression is visibly down-regulated, and this throughout the brain (see also Additional data file 8a-d). Upon amphetamine administration, $(\mathbf{f}, \mathbf{h})$ the expression of foxg $/$ and $/ h \times 8$ is reduced in the parvocellular preoptic nucleus, posterior part (PPp) (foxg I and Ihx8) and in the ventral zone of the periventricular hypothalamus (Hv) (foxgl), when compared to (e, g) the brains of animals injected with a saline solution. The expression pattern of these genes remains unchanged in other brain areas upon amphetamine administration (Additional data file $8 \mathrm{e}-\mathrm{h}$ ). Scale bars $=100 \mu \mathrm{m}$ in all panels. $\mathrm{D}=$ dorsal telencephalic area; $\mathrm{Hv}=$ ventral zone of the periventricular hypothalamus; $\mathrm{mt}=$ midline of the telencephalon; $\mathrm{PPp}=$ parvocellular preoptic nucleus, posterior part; $\mathrm{V}=$ ventral telencephalic area; $\mathrm{VL}=$ ventrolateral thalamic nucleus. 
slc6a5/glyT2 is increased in wild-type and excessively increased in nad animals upon amphetamine administration. Hence, chronic amphetamine administration may modify the amount of glycine at the synapse via Slc6a5 activity, with possible consequences on the development or reinforcement of amphetamine-triggered reward. In mammals, PYY antagonizes the orexigenic and anxiolytic effect of Neuropeptide $Y$, which can itself elicit CPP reward behavior [71]. We observed $p y y-b$ expression to be down-regulated by amphetamine in wild-type but not nad animals. Down-regulation of $p y y-b$ could reinforce the activity of Neuropeptide Y, thereby contributing to the development of reward, while its lack of response in nad animals might mediate the resistance of this mutant to CPP behavior.

Seven genes encoding proteins related to axonal or synaptic dynamics were also recovered in the reward pool (Additional data file 4). Among these, two belong to families that may be directly relevant to addiction or drug use: $D r$. 83111, encoding a protein highly similar to Neuregulin 1 and a Drebrin-like protein-encoding gene ( $\mathrm{Dr} .76820$ ). Neuregulin 1 signaling plays a prominent role in synapse plasticity in the mature brain by controlling excitatory and inhibitory synaptic transmission [72-74], which might underlie the propensity towards drug abuse [75]. In humans, it has also been identified as a susceptibility factor for schizophrenia, a disease often co-morbid with substance use disorder. We found that Neuregulin 1 was strongly up-regulated by amphetamine in wild-type animals, and massively down-regulated in nad. This differential response may play a role in the different CPP behavioral response of nad. Drebrin, an F-actin-binding protein enriched in dendritic spines, is essential for spine morphogenesis and activity-dependent synaptic targeting of NMDA receptors [76-79]. Both the morphology and density of dendritic spine in the ventral tegmental area, nucleus accumbens and motor cortex are altered by amphetamine and cocaine [80]. We found that expression of Drebrin is increased upon amphetamine administration in wild-type animals, but fails to be upregulated under the same conditions in nad mutants, suggesting that altered changes in dendritic spine remodeling accompany the resistance of nad to amphetamine.

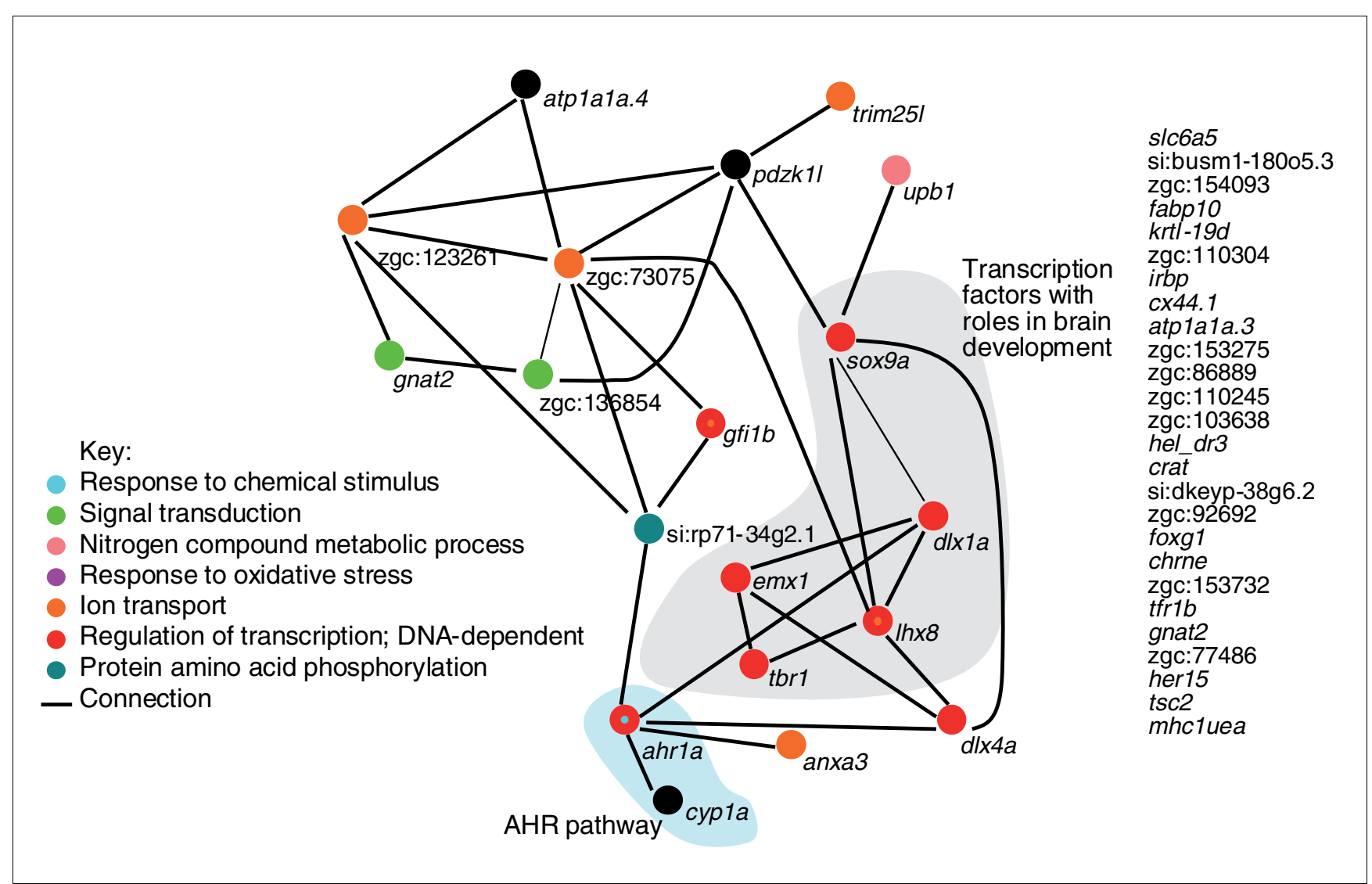

Figure 7

Network view of 18 genes from the reward pool functionally linked by the ZFISHDB database. Nodes are connected if functional interactions between the genes are provided by the cluster of orthologous genes (COG) mode of the STRING database. The GO terms listed are not exhaustive. The genes that have a mouse homologue and were thus included in the analysis, but which were not linked to other genes in the pool, are listed separately on the right. In addition, her/5, tsc2 and mhcluea, for which the program did not find suitable mouse homologues, but for which we manually checked for associates with the other genes in the network, are also listed. 
Genes encoding components of the dopamine pathway were not identified in our experiment, although amphetamine is considered to primarily increase extracellular dopamine levels in the forebrain $[81,82]$. In drug-addicted subjects, the concentration of dopamine receptors (namely D2) is lowered on the cell surface as revealed by imaging studies [83]. However, corresponding changes in gene expression have not been consistently reported, suggesting that the modulation of the dopamine pathway may not occur at the level of transcription [9]. Alternatively, microarray sensitivity may be insufficient to detect functionally relevant but small amplitude changes in the expression of weakly expressed factors such as dopamine signaling components [10]. In support of this, although our microarray chips contained a large representation of genes encoding transporters, receptors, and synthesis and metabolism enzymes for most neurotransmitters (including dopamine, glutamate, noradrenaline, 5-hydroxytryptamine, Neuropeptide $\mathrm{Y}$, acetylcholine, glycine and opiates), we only obtained reproducible hybridization, indicating sufficient expression, for a few of these genes (Additional data files 1 to 3 ). From these, apart from chnre and slc6a5 (discussed above), only glutamate transporter $5 A$ and neuropeptide $Y$ receptor $Y_{7}$ showed significantly modified expression upon chronic amphetamine exposure (an up-regulation in both cases). However, because similar changes were observed in nad mutants, these two genes were not present in the reward pool and so are unlikely to account for the non-development of CPP in nad.

\section{nad mutants highlight the importance of brain developmental transcription factors in the CPP response to amphetamine, and point to a link between amphetamine administration and the control of adult neurogenesis}

An exciting new contribution of our work is to highlight the importance of transcription factors implicated in development in the response to amphetamine-triggered reward. The category 'transcription' was further enriched in the reward pool over individual experiments and so was prominently revealed by our combined microarray strategy. The significance of transcription factors is further strengthened in that all genes classified under 'development' in this analysis are also transcription factors (Figure $3 \mathrm{~b}$ ), and is supported by several validations. Firstly, all chosen transcription factors of the reward pool tested by qPCR $(n=6)$ displayed changes in transcription levels upon amphetamine administration in wild-type animals and, in four cases, these changes were severe enough to be detected by in situ hybridization. The altered response of these four genes upon drug treatment in mutants compared to wild-type was also validated by qPCR. Secondly, several of these genes (Ahr1, Dlx1, Foxg1, Hes5, Sox9 and Tbr1) have been related to drug use or administration in mammals in other studies [62,67,84-87]. Finally, these genes appear to be functionally connected according to the ZFISHDB software; thus, they may participate in a common regulatory network. Strikingly, all these genes have rec- ognized roles during vertebrate brain development and also display persistent expression in the adult brain (Additional data file 6), including the mouse brain (see below), suggesting that their relevance for reward-induced behavior could be extended to adult mammals.

Together, these observations suggest that the development of CPP behavior may rely on the regulation of developmental genes that possibly maintain a modulatory role during adulthood, perhaps contributing to brain plasticity. Brain plasticity is thought to contribute to the learning of addictive behaviors and can underlie long-lasting changes mediating the persistent effects of addiction [88], and several recent reports show that embryonic factors can be recycled in the adult to regulate brain plasticity (for example, [89]). Thus, it is possible that the transcription factors identified in our reward pool are normally reused for modulatory or adaptive processes in the adult, and would here contribute to plasticity events triggered by the drug during the development of CPP. It will be crucial to test the function of these factors in the adult brain, also in the light of behavioral assays, to understand the functional relation between their functional regulation and the stepwise development of addiction behavior.

Classically, the modulatory events believed to underlie addiction involve synaptic or signaling plasticity. The gene regulation network we uncovered can also serve as a valuable entry point towards identifying further plasticity process(es) that might underlie the different behavioral effects of amphetamine in wild-type animals versus nad mutants. Our results highlighting developmental transcription factors suggest that fundamental cellular reconfigurations might also contribute to plasticity. In addition, expression of these factors in the mouse and fish brain, and functional assessments in mouse, all point towards a prominent role during neurogenesis. $d l_{1} 1 a / D x_{1}$ is expressed in the developing mouse ventral forebrain where it controls the formation of GABAergic neurons [36]. In the adult brain, it is involved in maintaining hippocampal interneurons [30]. Emx1 participates in the regionalization of the embryonic mouse cortex and the production of neuronal subtypes [37,38], and adult mice mutant for Emx1 exhibit impaired hippocampal neurogenesis [29]. Mouse $L h x 8$ is required for the development and maintenance of forebrain cholinergic neurons [32,39]. Mouse Sox9 is present in the stem cells of the peripheral and central nervous system, is essential for gliogenesis [90] and has also been isolated as a co-factor for proneural genes [91]. Tbr1 expression characterizes a freshly postmitotic state in the formation of glutamatergic pyramidal projection neurons of the developing mouse neocortex [41], and is maintained during adult hippocampal neurogenesis [33]. AhR (aryl hydrocarbon receptor) overexpression in developing neurons has been linked to premature differentiation [92]. Finally, although Her15 (and its mouse orthologue Hes5) and Foxg1 have not been connected to other transcription factors based on the literature co-citations used by our database, both genes are also expressed in embryonic 
neuroepithelial progenitors where they are involved in progenitor maintenance [93-96]. Later, FoxG1 is strongly expressed in areas of adult neurogenesis, including the subependymal zone of the lateral ventricle and the dentate gyrus of the hippocampus, and juvenile mice haploinsufficient for FoxG1 show impaired hippocampal neurogenesis [27]. Hes5 expression has also been described in astrocytes in neurogenic zones of the adult mouse brain [28]. Together, these data suggest that most of the transcription factors recovered in the reward pool are linked by their function or at least their expression at one or the other step of neurogenesis control, including in the adult brain. Although we do not yet have a complete account of zebrafish gene expression patterns at the single cell level, our observations are in agreement with this hypothesis in adult fish as well: all the transcription factorencoding genes investigated in this study have in common that they are expressed in all or part of the adult forebrain ventricular zone (Additional data file 6), which has been demonstrated to be neurogenic [97-101]. The expression profiles of her15 and $g$ fir $b$ are particularly striking due to their strict restriction to the ventricular zone (Additional data file $6 \mathrm{e}, \mathrm{f}$ ) and their massive down-regulation upon chronic amphetamine treatment (Figure 5). emx1, soxga and tbr1 are also noteworthy for their prominent expression in the neurogenic area of the lateral pallium (Additional data file 6c, I, arrows; and data not shown), an area thought to be the functional equivalent of the hippocampus [102,103].

A link between adult neurogenesis and drug abuse has been previously investigated, although with mixed results. Overall, the effect of amphetamine on proliferation during chronic application remains to be examined, although chronic cocaine use has been shown to decrease cell proliferation in the germinal zone of the adult mouse hippocampus [104,105] (for reviews, see $[105,106]$ ), while withdrawal from cocaine self-administration triggers accelerated maturation of adult newborn hippocampal neurons [107]. Given the postulated function of adult hippocampal neurogenesis in the acquisition and consolidation of memories (including their spatial and contextual components), these alterations could play a role in the cognitive processes associated with the development, reinforcement or relapse of addiction. Our results strongly suggest that amphetamine also triggers changes in adult neurogenesis (this paper, and KJW, unpublished observations), which might involve or result in the changes in transcription factor expression that we observed. It is now important to investigate this point in detail. Our experimental strategy relying on the lack of behavioral response of nad mutants further stresses that the regulation of these transcription factors might directly or indirectly link amphetamine and behavior. However, it seems unlikely that the development of CPP observed after 7 days, and which fails in nad mutants, could already result from an effect of amphetamine on adult neurogenesis. Newborn neurons require at least 3 weeks to be incorporated into active circuits in the adult mouse and our previous data suggest a similar time- frame in zebrafish [97]. It is possible, however, that rapid alterations of the ventricular zone by amphetamine could indirectly affect the physiology of neurons in the vicinity, for instance by altering the trophic support normally provided by ventricular radial glia cells leading to an effect on CPP. These changes could be modified in the nad mutant. Alternatively, modified neurogenesis upon amphetamine administration could account for later behavioral changes, a hypothesis that remains to be tested in our mutants.

\section{Conclusions}

Our experimental strategy based on the nad mutant, which fails to respond to amphetamine in the CPP test, allowed the first identification of a subset of amphetamine-regulated transcripts linked to the reward response. This pool contains gene categories previously linked to the use of addictive drugs, thereby validating our data. Enrichment analyses, confirmed by qPCR and in situ hybridization, highlighted a set of genes encoding transcription factors within this pool, most of which are involved in brain development, and which can partially be organized into a network of functional interactions. Together, we propose that the re-use of a developmental transcription factor-mediated network accompanies or underlies the behavioral response to amphetamine in the adult brain. Some of these factors, expressed in adult neurogenic domains and dramatically down-regulated by amphetamine, can further serve as valuable new entry points into studying the link between neurogenesis and addiction.

\section{Materials and methods \\ Animals and maintenance}

Adult zebrafish were kept in the fish facility as described in Kimmel et al. [108]. For practical reasons (ease of intraperitoneal injections) all experiments were performed on females. In preliminary experiments, we did not notice any difference in the response of males and females to D-amphetamine for a given genotype [21]. Throughout the experiment, care was taken to perform procedures involving animals, such as place preference measurements and injections, at the same time of the day. Mutagenesis, mutant screening and array experiments involving mutants and siblings were performed on fish of the $\mathrm{AB}$ background. Mutant fish were maintained in this background throughout the study. Behavioral experiments on wild-type fish were conducted on an intercross background between $\mathrm{AB}$ and Tübingen (Tü). The $\mathrm{AB}$ and Tü lines are both wild-type lines. $\mathrm{AB}$ fish have been inbred over more than 100 generations to date [26], and Tü fish over approximately 25 generations. Both lines, as well as their common progeny (AB/Tü), are sensitive to amphetamine with a similar dose-response and perform equally well in the CPP test [21]. They have been used here for reasons of availability. 


\section{ENU mutagenesis and screening for dominant mutations affecting reward}

Adult males of the $\mathrm{AB}$ strain were subject, over a 4-week interval, to four 1-hour incubations in $3 \mathrm{mM}$ ENU. Three weeks after this treatment, F1 animals were generated by pair-wise mating of ENU mutagenized males with $\mathrm{AB}$ females. The specific locus rate at this stage was estimated to be $1 / 670$ against the golden (slc24a5) locus. Three- to ninemonth-old $\mathrm{F} 1$ animals were screened for their change in place preference in response to $40 \mu \mathrm{g} / \mathrm{g} \mathrm{D}$-amphetamine (throughout the text referred to as amphetamine), as described below. Of the $396 \mathrm{~F} 1$ adults that were screened, 4 failed to respond to amphetamine, although they exhibited normal place preference without drug and, hence, could recognize the visual cues of the test tank. They also displayed normal amphetamine content in the brain after injection, as measured using denaturing high-performance liquid chromatography (not shown). These animals were considered potential dominant amphetamine-resistant mutants and were crossed against wild-type $\mathrm{AB}$ fish to test for transmission of the phenotype. The behavior of 20 F2 adults from these crosses was again assessed in the CPP test in response to amphetamine. For one of these four $\mathrm{F} 1$ candidate mutants, 50\% non-responders were obtained in the F2 and all further generations, arguing for a bona fide dominant mutation. We refer to this mutation as naddne 3256 .

\section{Behavioral assays}

The CPP experiment was performed according to Ninkovic and Bally-Cuif [21]. Briefly, the fish were habituated to a biased two-part chamber (days 1 to 2), followed by the determination of the initial place preference (day 3). Subsequently, for test animals, amphetamine injections (40 $\mu \mathrm{M}$; days 4,6 and 8) were paired with the initially non-preferred side of the chamber, and control injections of saline solution (days 5 and 7) were paired with the initially preferred side. Control animals are injected with saline every day but likewise paired with the initially non-preferred side on days 4, 6 and 8 and with the initially preferred side on days 5 and 7 . On day 9 the final place preference was measured. Conditioning was estimated as in Ninkovic and Bally-Cuif [21] as the change in place preference before and after treatment, relative to the place preference before treatment. Within a mutant family, fish were designated as mutant (mut) when there was no change or a negative change in place preference after amphetamine administration. Fish were designated as wild-type siblings (sib) when the percentage of change was higher than $5 \%$. If the percentage of change was between 0 and $5 \%$, the fish were not included in the microarray analysis in order to avoid incorrect phenotyping.

\section{Microarray study design and data analysis}

The experimental design of this study was aimed at the identification of genes that respond differently to amphetamine treatment in wild-type zebrafish and the reward mutant nad. In order to identify the signature gene set for the interaction term genotype ${ }^{*}$ amphetamine treatment, we examined three biological contrasts: the response of wild type (wt) on amphetamine (experiment 1); the differential gene expression in the presence of amphetamine between wild type and mutant (experiment 2); and the base line difference in transcription between wild type and mutant (experiment 3). The animals used in the different experiments were all aged between 6 and 12 months and were manipulated as follows. Experiment 1: $\mathrm{wt}+, \mathrm{AB}$ fish subjected to the CPP behavioral assay and sacrificed 30 minutes after the final amphetamine injection (the day after place preference determination; day 10); wt-, AB control fish of the CPP behavioral assay (with control saline injections at the same time points as the wt+ fish were injected with amphetamine). Experiment 2: mut+, $\mathrm{AB}$ animals from a nad family of the $\mathrm{F} 6$ generation (obtained from pairing a $\mathrm{nad} /+\mathrm{F}_{5}$ heterozygote fish and an $\mathrm{AB}$ fish) identified as mutant based on the CPP assay and sacrificed 30 minutes after the final amphetamine injection on day 10; mut-, siblings identified as wild type in the same experiment, and treated exactly the same as the mut+ fish with regard to amphetamine administration. Experiment 3: mut-, AB animals from a 55 nad family identified as mutant in the CPP test and left without drug for 2 months afterwards; sib-, siblings identified as wild type in the same experiment and left without drug for 2 months.

One-color Agilent microarray experiments were performed using three biological replicates for each condition. Each replicate contained the RNA from four to five pooled brains. Microarray data were imported into Rosetta Resolver 7.1 (Rosetta Biosoftware, Seattle, Washington, USA) and analyzed using the Rosetta error model for gene expression analysis. The Resolver system calculates ratios for the Agilent intensity microarrays by combining all pairwise ratios of the individual sequence data making up the numerator (for example, treatment replicates) against those making up the denominator (for example, the control replicates) of the specified ratio. The calculations begin with scaling the intensity signals for each sequence relative to the average intensity signal of the entire array. For each sequence, the two scaled intensity values for each pairwise ratio are then converted to the logarithmic scale and the averaging and ratio computation is performed on the logarithms. The sequence errors are accordingly propagated through the log-transformation and averaging. This propagated error is used to determine the statistical significance of the final logarithm of the ratios, that is, the $P$-values corresponding to differential expression are calculated based on the $\log$ (ratio). In the Rosetta analysis, first our microarray data were subjected to default intensity error modeling and results from triplicate experiments were combined using the default intensity experiment builder. Next, ratio experiments were built from the intensity data using the Agilent/Intensity-pairwise ratio builder with the control group (salt for experiment 1 and wild type for experiments 2 and 3) as baseline. Data were analyzed at the level of UniGene clusters (UniGene build \#105). The resulting $P$-values from 
the Rosetta error model for gene expression analysis were adjusted for multiple testing using the method of Benjamini and Hochberg to control the false discovery rate. $P$-values were corrected with the p.adjust function in $\mathrm{R}$ statistical software. The significance cut-offs were set at $P<0.01$ (Benjamini and Hochberg adjusted $P<0.1$ ) and absolute fold change $>1.5$. The false discovery rates at the initial $P$-value cut-off of < 0.01 are, for each experiment: experiment 1 , < 0.0816; experiment 2, < 0.07302 ; experiment $3,<0.0504$. Venn diagrams were constructed using the 'compare biosets' function of Rosetta resolver.

\section{RNA extraction and microarray study design}

Total RNA was extracted from whole brains using RNeasy Mini Kit (Qiagen GmbH, Hilden, Germany) following the protocol 'Purification of Total RNA from Animal Tissues'. For the disruption and homogenization step, brains were dissected and immediately frozen in liquid nitrogen. Then, 600 $\mu \mathrm{l}$ buffer RLT was added to each brain and the tissue was homogenized using a needle and syringe. The samples were individually controlled for RNA quality and genomic contamination using 2100 Bioanalyzer (Agilent Technologies, Palo Alto, CA, USA), according to the manufacturer's instructions. Samples from four to five brains were then pooled to generate a single replicate. The animals used in the different experiments were all aged between 6 and 12 months.

\section{RNA amplification, labeling and hybridization}

The RNA samples were amplified with the Agilent Low Input Linear Amplification kit PLUS, One Color (Agilent Technologies). The labeling, hybridization and data extraction were performed at ServiceXS (Leiden, The Netherlands). Briefly, 500 ng total RNA in an $8.3 \mu$ l volume was mixed with $1.2 \mu \mathrm{l}$ of T7 promoter primer. Primer and template were denatured by incubating at $65^{\circ} \mathrm{C}$ for 10 minutes and annealed by placing the reaction on ice. The first strand reaction was performed by adding a master mix containing $5 \times$ First Strand Buffer, dithiothreitol, $10 \mathrm{mM}$ dNTP mix, RNaseOUT, and Moloney murine leukemia virus reverse transcriptase, and incubated at $40^{\circ} \mathrm{C}$ for 2 hours. The Moloney murine leukemia virus reverse transcriptase was inactivated by incubation at $65^{\circ} \mathrm{C}$ for 15 minutes and the samples were directly transferred to ice. Samples were labeled by adding $2.4 \mu$ l cyanine 3 -CTP. In vitro transcription was initiated by addition of the IVT Mastermix containing $4 \times$ transcription buffer, dithiothreitol, NTP mix, 50\% polyethylene glycol, RNaseOUT, inorganic pyrophosphates, T7 RNA polymerase and incubated at $40^{\circ} \mathrm{C}$ for 2 hours. Qiagen RNeasy mini spin columns were used for purification of the labeled cRNA as described in the Agilent user manual. After amplification and purification, the samples were checked for RNA concentration and dye incorporation on the Nanodrop ND-10oo by using $1 \mu \mathrm{l}$ of the $60 \mu \mathrm{l}$ elution solution (nuclease-free water). Hybridization and washing was performed using the standard Agilent protocol.
The microarray slides were custom designed by Agilent Technologies. The slides contained, in total, 43,371 probes each 60 oligonucleotides long. Of these probes, a total of 21,496 were identical to the probes present on the Agilent probe set that is commercially available under catalog number 013223_D. Most of the additional probes were designed using the eArray software from Agilent Technologies [109]. Settings used were the following: base composition methodology, best probe methodology and design with 3 ' bias. The Agilent Danio rerio transcriptome was used as a reference database. A small number of probes were manually designed in order to obtain gene-specific probes for members of larger gene families. The complete design of the microarrays has been submitted to the Gene Expression Omnibus (GEO) database, under the platform submission number [GEO:GPL7735].

\section{Microarray imaging and data analysis}

Scanning of the microarray slides was performed using the Agilent dual laser DNA microarray scanner. The microarray data were processed from raw data image files with Feature Extraction Software version 9.1, protocol GE1-v1_91(Agilent Technologies). The microarray slides were custom designed by Agilent Technologies as described in Stockhammer et al. [110]. Unigene lists of individual experiments can be found in Additional data files 1 to 3 . All microarray data were submitted to the GEO database [GEO:GSE14399].

\section{Gene Ontology term enrichment analysis}

GO term enrichment analysis was performed on differentially expressed genes $(P<0.01$; fold change $<-1.5$ or $>1.5)$ from the individual experiments, as well as on the reward pool. Lists of differentially expressed genes were imported into Pathway Studio (Ariadne Genomics, Rockville, MD, USA). Before analysis the ResNet 5.0 database of this software was extended to include the zebrafish protein annotation. The Pathway Studio program determines the human, rat and mouse orthologues of the zebrafish transcripts, using the BLAST best reciprocal hit method. This information is used to perform the enrichment analysis, in which Pathway Studio calculates the statistical significance of the overlap between the input list and a GO group by applying Fisher's exact test. The resulting $P$ value depends on the extent of overlap between the input list and a group as well as the sizes of the list and a group. In addition, we performed a Bonferroni correction for $P$-values calculated from Fisher's exact test by using the p.adjust function in the package 'stats' of the statistical software R [111]. We considered a GO term to be statistically significant if the corresponding adjusted $P$-value is $<0.05$.

\section{Assessment of the functional interactions between recovered genes}

Functional interactions between zebrafish genes in the reward pool were inferred from the STRING database [112]. STRING integrates and scores information derived from high-throughput experiments, genomic context, and previous knowledge such as text-mining of abstracts. For zebrafish, the 
number of interactions is small compared to better annotated species like mouse or human. In order to enrich the interactions in our gene set, we transferred interactions from orthologous genes as provided by the cluster of orthologous genes mode of STRING, where the information of orthologous groups of proteins relies on an extended version of the COG database [113]. The ZebraFish Interaction SearcH DataBase (ZFISHDB) integrates all interactions between clusters of orthologous genes, relying on STRING 7.0. It allows the input of a set of fish genes and outputs interactions between those genes with a STRING combined score above o.8. In addition, ZFISHDB offers a gene ontology filter to reduce the size of large data sets. It is publicly available [114]. Genes without mouse homologues are not considered by the present version of the database.

\section{Quantitative real-time PCR}

Total RNA was extracted from whole brains using the RNeasy Mini Kit (Qiagen). The qPCR experiments and the statistical analysis were performed using the LightCycler 1.2 system (Roche, Basel, Switzerland) and the relative expression software tool REST [115] as previously described [116]. Briefly, the statistical model used by this software is a pair-wise fixed reallocation randomization test. The software returns the probability of the alternative hypothesis $(P(H 1))$, which is that the difference between sample and control groups is due only to chance. For each test 50,000 randomization iterations were applied and an associated probability ( $P$-value) of $P<$ 0.05 was considered significant. Real time PCR experiments were performed in replicates of eight. The list of used primers and probes is provided in Additional data file 9.

\section{In situ hybridization}

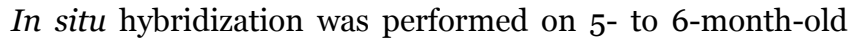
$\mathrm{AB} / \mathrm{Tü}$ fish that had either been treated with amphetamine $(40 \mu \mathrm{M})$ or saline solution, once a day, for 18 days. Animals were sacrificed and the brains were removed after fixation in 4\% paraformaldehyde. Dissected brains were then postfixed in $4 \%$ paraformaldehyde overnight. The brains were then embedded in albumin-gelatine:sucrose denatured with glutaraldehyde. Cross-sections of $70 \mu \mathrm{m}$ were made using a vibratome, after which the sections were washed in PBS-0.1\% Tween-20 and dehydrated through a methanol series. In situ hybridization was performed according to published protocols [117] for whole-mount embryos, followed by staining for alkaline phosphatase activity using Nitro-Blue Tetrazolium (NBT)- 5-Bromo-4-Chloro-3-Indolyl-Phosphate (BCIP) (Roche, Basel, Switzerland). Initially, one brain was used per treatment. All sections were photographed and corresponding sections were compared between treatments. The in situ hybridization for genes, the expression patterns of which showed a visible difference between amphetamine treatment and control, was repeated once. Sections were photographed with an Axioplan2 stereomicroscope and processed using the Axiovision 4.1 software (Zeiss). To generate probes, partial cDNAs for the genes of interest were cloned from PCR prod- ucts (PCR conditions available upon request; PCR primers provided in Additional data file 10) into pCRII-TOPO (Invitrogen, Karlsruhe, Germany); for her15) or pSC-A-amp/kan using the StrataClone PCR Cloning Kit (Stratagene, La Jolla, CA, USA); for all other genes) following the manufacturers' instructions. All clones were verified by sequencing. The RNA probes were synthesized following published protocols [117].

\section{Abbreviations}

CPP: conditioned place preference; ENU: N-ethyl-N-nitrosourea; GEO: Gene Expression Omnibus; GO: Gene Ontology; NBT: Nitro-Blue Tetrazolium; qPCR: quantitative PCR.

\section{Authors' contributions}

KJW designed and performed the experiments and wrote the manuscript (with LB-C), WN performed mutant identification experiments and worked on the manuscript, JN conducted the behavior screen and identified the nad mutant, ST provided technical assistance for in situ hybridizations, DT performed the Pathway Studio analyses in WW's lab, AHM and HPS designed the arrays and helped with their interpretation, DH, CM and FT designed the ZFISHDB database, and LB-C directed the work, designed the experiments and wrote the manuscript (with KJW).

\section{Additional data files}

The following additional data are available with the online version of this paper: a complete Unigene list of genes recovered in microarray experiment 1 'wt+/wt-' (Additional data file 1); a complete Unigene list of genes recovered in microarray experiment 2 'mut+/sib+' (Additional data file 2); a complete Unigene list of genes recovered in microarray experiment 3 'mut-/sib-' (Additional data file 3); a list of the 139 genes contained in the reward pool (Additional data file 4); a list of enriched GO terms in individual experiments (Additional data file 5); figures showing expression in the adult telencephalon for ten transcripts chosen for validation (Additional data file 6); qPCR data for validated transcripts in conditions of chronic and acute amphetamine administration (Additional data file 7); figures showing expression of her15, $g$ fir $b$, foxg 1 and $l h x 8$ in the adult brain upon amphetamine administration (Additional data file 8); a list of the primers and probes used for qPCR (Additional data file 9); a list of the primers used to clone in situ hybridization probes (Additional data file 10).

\section{Acknowledgements}

We are grateful to all past and present members of the Bally-Cuif lab for their helpful suggestions, and in particular to B Hesl and A Folchert for their initial help with mutant screening. Thanks also to $C$ Goridis and $P$ Vernier for their critical reading of the manuscript, and to B Kieffer for stimulating comments on this work. We also acknowledge the Neuherberg fish facility crew for expert fish care. Thanks to R Arnold, T Rattei and F Buggenthin for their help in database development. Thanks to A Zakrzewska for help 
with GEO submission. Thanks to A Mayerhofer for helpful discussions. This work was supported by grants from the VolkswagenStiftung (to LB-C), the Life Science Stiftung (contract GSF2005/0I; to LB-C), the Center for Protein Science Munich (CIPSM; to LB-C), the ICM (to LB-C), the European Community (FP6 IP ZF-Models, contract LSHC-CT-2003-503466; to LB-C and HS), the Helmholtz Alliance on Systems Biology (project CoReNe; to CM. and FT) and the BMBF (NGFN program; to WW).

\section{References}

I. Office of National Drug Control Policy (ONDCP): The Economic Costs of Drug Abuse in the United States 1992 -1998 Publication NCJ-190636. Washington, DC: Office of National Drug Control Policy; 200I.

2. Wise RA: Brain reward circuitry: insights from unsensed incentives. Neuron 2002, 36:229-240.

3. Vanderschuren LJ, Everitt BJ: Drug seeking becomes compulsive after prolonged cocaine self-administration. Science 2004, 305:1017-1019.

4. Bossert JM, Ghitza UE, Lu L, Epstein DH, Shaham Y: Neurobiology of relapse to heroin and cocaine seeking: an update and clinical implications. Eur J Pharmacol 2005, 526:36-50.

5. Kelley AE: Memory and addiction: shared neural circuitry and molecular mechanisms. Neuron 2004, 44:161-179.

6. Mash DC, ffrench-Mullen J, Adi N, Qin Y, Buck A, Pablo J: Gene expression in human hippocampus from cocaine abusers identifies genes which regulate extracellular matrix remodeling. PLOS ONE 2007, 2:e I I87.

7. Zhang D, Zhang L, Tang Y, Zhang Q, Lou D, Sharp FR, Zhang J, Xu M: Repeated cocaine administration induces gene expression changes through the dopamine DI receptors. Neuropsychopharmacology 2005, 30: I443-1454.

8. Yamamoto $H$, Imai K, Takamatsu $Y$, Kamegaya E, Hara $Y$, Shimada K, Yamamoto T, Shen HW, Hagino Y, Kobayashi H, Ide S, Sora I, Koga $\mathrm{H}$, Ikedaa $\mathrm{K}$ : Changes in expression of the mouse homologues of KIAA genes after subchronic methamphetamine treatment. Ann N Y Acad Sci 2004, 1025:92-I0I.

9. Rhodes JS, Crabbe JC: Gene expression induced by drugs of abuse. Curr Opin Pharmacol 2005, 5:26-33.

10. Yuferov V, Nielsen D, Butelman E, Kreek MJ: Microarray studies of psychostimulant-induced changes in gene expression. Addict Biol 2005, 10: 101-1 18.

11. Schultz W: Getting formal with dopamine and reward. Neuron 2002, 36:24I-263.

12. Lehrmann E, Colantuoni C, Deep-Soboslay A, Becker KG, Lowe R, Huestis MA, Hyde TM, Kleinman JE, Freed WJ: Transcriptional changes common to human cocaine, cannabis and phencyclidine abuse. PLOS ONE 2006, I:el I4.

13. Winstanley CA, LaPlant Q, Theobald DE, Green TA, Bachtell RK, Perrotti LI, DiLeone RJ, Russo SJ, Garth WJ, Self DW, Nestler EJ: DeltaFos $B$ induction in orbitofrontal cortex mediates tolerance to cocaine-induced cognitive dysfunction. J Neurosci 2007, 27:10497-10507.

14. Bibb JA, Chen J, Taylor JR, Svenningsson P, Nishi A, Snyder GL, Yan Z, Sagawa ZK, Ouimet CC, Nairn AC, Nestler EJ, Greengard P: Effects of chronic exposure to cocaine are regulated by the neuronal protein Cdk5. Nature 200I, 41 0:376-380.

15. Lett BT, Grant VL: The hedonic effects of amphetamine and pentobarbital in goldfish. Pharmacol Biochem Behav 1989, 32:355-356.

16. Levens N, Akins CK: Cocaine induces conditioned place preference and increases locomotor activity in male Japanese quail. Pharmacol Biochem Behav 200I, 68:7I-80.

17. Ninkovic J, Folchert A, Makhankov YV, Neuhauss SC, Sillaber I, Straehle U, Bally-Cuif L: Genetic identification of AChE as a positive modulator of addiction to the psychostimulant $D$ amphetamine in zebrafish. J Neurobiol 2006, 66:463-475.

18. Bretaud S, Li Q, Lockwood BL, Kobayashi K, Lin E, Guo S: A choice behavior for morphine reveals experience-dependent drug preference and underlying neural substrates in developing larval zebrafish. Neuroscience 2007, 146:1 109-1116.

19. Sulzer D, Edwards RH: Antidepressants and the monoamine masquerade. Neuron 2005, 46:1-2.

20. Tzschentke TM: Measuring reward with the conditioned place preference (CPP) paradigm: update of the last decade. Addict Biol 2007, I 2:227-462.

21. Ninkovic J, Bally-Cuif $L$ : The zebrafish as a model system for assessing the reinforcing properties of drugs of abuse. Methods 2006, 39:262-274.

22. Crabbe JC: Genetic contributions to addiction. Annu Rev Psychol 2002, 53:435-462.

23. Volkow ND, Li TK: Drug addiction: the neurobiology of behaviour gone awry. Nat Rev Neurosci 2004, 5:963-970.

24. Everitt BJ, Belin D, Economidou D, Pelloux Y, Dalley JW, Robbins TW: Review. Neural mechanisms underlying the vulnerability to develop compulsive drug-seeking habits and addiction. Philos Trans R Soc Lond B Biol Sci 2008, 363:3 I25-3I 35.

25. Ensembl [http://www.ensembl.org/index.html]

26. ZFIN [http://zfin.org/cgi-bin/webdriver?Mlval=aa-ZDB_home.apg]

27. Shen L, Nam HS, Song P, Moore H, Anderson SA: FoxGI haploinsufficiency results in impaired neurogenesis in the postnatal hippocampus and contextual memory deficits. Hippocampus 2006, 16:875-890.

28. Ohtsuka T, Imayoshi I, Shimojo H, Nishi E, Kageyama R, McConnell SK: Visualization of embryonic neural stem cells using Hes promoters in transgenic mice. Mol Cell Neurosci 2005, 31:109-122

29. Hong SM, Liu Z, Fan Y, Neumann M, Won SJ, Lac D, Lum X, Weinstein PR, Liu J: Reduced hippocampal neurogenesis and skill reaching performance in adult Emx I mutant mice. Exp Neurol 2007, 206:24-32.

30. Cobos I, Calcagnotto ME, Vilaythong AJ, Thwin MT, Noebels JL, Baraban SC, Rubenstein JL: Mice lacking DlxI show subtype-specific loss of interneurons, reduced inhibition and epilepsy. Nat Neurosci 2005, 8: 1059-1068.

3I. Tsuda H, Jafar-Nejad H, Patel AJ, Sun Y, Chen HK, Rose MF, Venken $\mathrm{KJ}$, Botas J, Orr HT, Bellen HJ, Zoghbi HY: The AXH domain of Ataxin-I mediates neurodegeneration through its interaction with Gfi-I/Senseless proteins. Cell 2005, I 22:633-644.

32. Zhao $Y$, Marin O, Hermesz E, Powell A, Flames N, Palkovits M, Rubenstein JL, Westphal H: The LIM-homeobox gene Lhx8 is required for the development of many cholinergic neurons in the mouse forebrain. Proc Natl Acad Sci USA 2003, 100:9005-9010.

33. Hevner RF, Hodge RD, Daza RA, Englund C: Transcription factors in glutamatergic neurogenesis: conserved programs in neocortex, cerebellum, and adult hippocampus. Neurosci Res 2006, 55:223-233.

34. Huang P, Ceccatelli S, Hoegberg P, Sten Shi TJ, Hakansson H, Rannug $A$ : TCDD-induced expression of $A h$ receptor responsive genes in the pituitary and brain of cellular retinol-binding protein (CRBP-I) knockout mice. Toxicol Appl Pharmacol 2003, 192:262-274.

35. Allen Atlas [http://www.brain-map.org/]

36. Marin O, Anderson SA, Rubenstein JL: Origin and molecular specification of striatal interneurons. J Neurosci 2000, 20:6063-6076.

37. Gorski JA, Talley T, Qiu M, Puelles L, Rubenstein JL, Jones KR: Cortical excitatory neurons and glia, but not GABAergic neurons, are produced in the EmxI-expressing lineage. J Neurosci 2002, 22:6309-63।4.

38. Shinozaki K, Miyagi T, Yoshida M, Miyata T, Ogawa M, Aizawa S, Suda $Y$ : Absence of Cajal-Retzius cells and subplate neurons associated with defects of tangential cell migration from ganglionic eminence in EmxI/2 double mutant cerebral cortex. Development 2002, I 29:3479-3492.

39. Mori T, Yuxing Z, Takaki H, Takeuchi M, Iseki K, Hagino S, Kitanaka J, Takemura M, Misawa H, lkawa M, Okabe M, Wanaka A: The LIM homeobox gene, L3/Lhx8, is necessary for proper development of basal forebrain cholinergic neurons. Eur J Neurosci 2004, | 9:3|29-3|4|.

40. Pompolo S, Harley VR: Localisation of the SRY-related HMG box protein, SOX9, in rodent brain. Brain Res 200I, 906: $143-148$.

4I. Englund C, Fink A, Lau C, Pham D, Daza RA, Bulfone A, Kowalczyk T, Hevner RF: Pax6, Tbr2, and Tbrl are expressed sequentially by radial glia, intermediate progenitor cells, and postmitotic neurons in developing neocortex. J Neurosci 2005, 25:247-25I.

42. Moroy $\mathrm{T}$ : The zinc finger transcription factor Growth factor independence I (Gfil). Int J Biochem Cell Biol 2005, 37:54 I-546.

43. Ellies DL, Stock DW, Hatch G, Giroux G, Weiss KM, Ekker M: Relationship between the genomic organization and the overlapping embryonic expression patterns of the zebrafish dlx genes. Genomics 1997, 45:580-590.

44. Kawahara A, Dawid IB: Developmental expression of zebrafish emxI during early embryogenesis. Gene Expr Patterns 2002, 
2:201-206.

45. Mueller T, Wullimann MF, Guo S: Early teleostean basal ganglia development visualized by zebrafish DIx2a, Lhx6, Lhx7, Tbr2 (eomesa), and GAD67 gene expression. J Comp Neurol 2008, 507:1245-1257.

46. Chiang EF, Pai Cl, Wyatt M, Yan YL, Postlethwait J, Chung B: Two sox9 genes on duplicated zebrafish chromosomes: expression of similar transcription activators in distinct sites. Dev Biol 200I, 23 I: I49-I63.

47. Miyake $\mathrm{A}$, Nakayama $\mathrm{Y}$, Konishi M, Itoh $\mathrm{N}$ : Fgf $\mathbf{9}$ regulated by $\mathbf{H h}$ signaling is required for zebrafish forebrain development. Dev Biol 2005, 288:259-275.

48. Stigloher C, Ninkovic J, Laplante M, Geling A, Tannhauser B, Topp S, Kikuta H, Becker TS, Houart C, Bally-Cuif L: Segregation of telencephalic and eye-field identities inside the zebrafish forebrain territory is controlled by Rx3. Development 2006, 133:2925-2935.

49. Sokolov BP, Polesskaya OO, Uhl GR: Mouse brain gene expression changes after acute and chronic amphetamine. J Neurochem 2003, 84:244-252.

50. Lau B, Bretaud S, Huang Y, Lin E, Guo S: Dissociation of food and opiate preference by a genetic mutation in zebrafish. Genes Brain Behav 2006, 5:497-505.

5I. Korostynski M, Piechota M, Kaminska D, Solecki W, Przewlocki R: Morphine effects on striatal transcriptome in mice. Genome Biol 2007, 8:RI28.

52. Del Arco A, Gonzalez-Mora JL, Armas VR, Mora F: Amphetamine increases the extracellular concentration of glutamate in striatum of the awake rat: involvement of high affinity transporter mechanisms. Neuropharmacology 1999, 38:943-954.

53. Florin SM, Kuczenski R, Segal DS: Regional extracellular norepinephrine responses to amphetamine and cocaine and effects of clonidine pretreatment. Brain Res 1994, 654:53-62.

54. Kily LJ, Cowe YC, Hussain O, Patel S, McElwaine S, Cotter FE, Brennan $\mathrm{CH}$ : Gene expression changes in a zebrafish model of drug dependency suggest conservation of neuro-adaptation pathways. J Exp Biol 2008, 21 I:1623-1634.

55. Ninkovic J, Folchert A, Makhankov YV, Neuhauss SC, Sillaber I, Straehle $U$, Bally-Cuif $L$ : Genetic identification of $A C h E$ as a positive modulator of addiction to the psychostimulant Damphetamine in zebrafish. J Neurobiol 2006, 66:463-475.

56. Gerlai R, Lahav M, Guo S, Rosenthal A: Drinks like a fish: zebra fish (Danio rerio) as a behavior genetic model to study alcohol effects. Pharmacol Biochem Behav 2000, 67:773-782.

57. Rink E, Wullimann MF: The teleostean (zebrafish) dopaminergic system ascending to the subpallium (striatum) is located in the basal diencephalon (posterior tuberculum). Brain Res 200I, 889:316-330.

58. Cozzi B, Viglietti-Panzica C, Aste N, Panzica GC: The serotoninergic system in the brain of the Japanese quail. An immunohistochemical study. Cell Tissue Res I99I, 263:27|-284.

59. Challet E, Miceli D, Pierre J, Reperant J, Masicotte G, Herbin M, Vesselkin NP: Distribution of serotonin-immunoreactivity in the brain of the pigeon (Columba livia). Anat Embryol (Berl) 1996, 193:209-227.

60. Rodrigues SL, Maseko BC, lhunwo AO, Fuxe K, Manger PR: Nuclear organization and morphology of serotonergic neurons in the brain of the Nile crocodile, Crocodylus niloticus. J Chem Neuroanat 2008, 35: I33-I45.

61. Kaslin J, Panula P: Comparative anatomy of the histaminergic and other aminergic systems in zebrafish (Danio rerio). J Comp Neurol 200I, 440:342-377.

62. Sokolov B, Polesskaya O, Uhl G: Mouse brain gene expression changes after acute and chronic amphetamine. J Neurochem 2003, 84:244-252.

63. Zhang D, Zhang L, Tang Y, Zhang Q, Lou D, Sharp F, Zhang J, Xu M: Repeated cocaine administration induces gene expression changes through the dopamine DI receptors. Neuropsychopharmacology 2005, 30: 1443-1454.

64. Lehrmann E, Colantuoni C, Deep-Soboslay A, Becker KG, Lowe R, Huestis MA, Hyde TM, Kleinman JE, Freed WJ: Transcriptionla changes common to human cocaine, cannabis and phenylcyclidine abuse. PLoS One 2006, I: I-I | 4 .

65. Mash DC, Ffrench-Mullen J, Adi N, Qin Y, Buck A, Pablo J: Gene expression in human hippocampus from cocaine abusers identifies genes which regulate extracellular matrix remodeling. PLoS One 2007, I I: I-1 I 87.

66. Sundstrom G, Larsson TA, Brenner S, Venkatesh B, Larhammar D:
Evolution of the neuropeptide $Y$ family: new genes by chromosome duplications in early vertebrates and in teleost fishes. Gen Comp Endocrinol 2008, I 55:705-716.

67. Kerns RT, Ravindranathan A, Hassan S, Cage MP, York T, Sikela JM, Williams RW, Miles MF: Ethanol-responsive brain region expression networks: implications for behavioral responses to acute ethanol in DBA/2J versus C57BL/6J mice. J Neurosci 2005, 25:2255-2266.

68. Feltenstein MW, See RE: The neurocircuitry of addiction: an overview. Br J Pharmacol 2008, 154:26I-274.

69. Gass JT, Olive MF: Glutamatergic substrates of drug addiction and alcoholism. Biochem Pharmacol 2008, 75:218-265.

70. Kalivas PW: Neurobiology of cocaine addiction: implications for new pharmacotherapy. Am J Addict 2007, 16:71-78.

7I. Brown CM, Coscina DV, Fletcher PJ: The rewarding properties of neuropeptide $\mathbf{Y}$ in perifornical hypothalamus vs. nucleus accumbens. Peptides 2000, $21: 1279-1287$.

72. Mei L, Xiong WC: Neuregulin I in neural development, synaptic plasticity and schizophrenia. Nat Rev Neurosci 2008, 9:437-452.

73. Britsch $\mathrm{S}$ : The neuregulin-I/ErbB signaling system in development and disease. Adv Anat Embryol Cell Biol 2007, 190:1-65.

74. Zhong C, Du C, Hancock M, Mertz M, Talmage DA, Role LW: Presynaptic type III neuregulin I is required for sustained enhancement of hippocampal transmission by nicotine and for axonal targeting of alpha7 nicotinic acetylcholine receptors. J Neurosci 2008, 28:91।I-9116.

75. Coyle JT: Substance use disorders and schizophrenia: a question of shared glutamatergic mechanisms. Neurotox Res 2006, 10:221-233

76. Takahashi H, Sekino Y, Tanaka S, Mizui T, Kishi S, Shirao T: Drebrindependent actin clustering in dendritic filopodia governs synaptic targeting of postsynaptic density-95 and dendritic spine morphogenesis. J Neurosci 2003, 23:6586-6595.

77. Takahashi H, Mizui T, Shirao T: Down-regulation of drebrin A expression suppresses synaptic targeting of NMDA receptors in developing hippocampal neurones. J Neurochem 2006, 97(SuppI I): II0-II5.

78. Lippman J, Dunaevsky A: Dendritic spine morphogenesis and plasticity. J Neurobiol 2005, 64:47-57.

79. Sekino Y, Kojima N, Shirao T: Role of actin cytoskeleton in dendritic spine morphogenesis. Neurochem Int 2007, 5 I:92-104.

80. Robinson TE, Kolb B: Alterations in the morphology of dendrites and dendritic spines in the nucleus accumbens and prefrontal cortex following repeated treatment with amphetamine or cocaine. Eur J Neurosci 1999, I I:1598-1604.

81. Williams JM, Galli A: The dopamine transporter: a vigilant border control for psychostimulant action. Handb Exp Pharmacol 2006:215-232

82. Riddle EL, Fleckenstein AE, Hanson GR: Role of monoamine transporters in mediating psychostimulant effects. Aaps J 2005, 7:E847-85I

83. Volkow ND, Fowler JS, Wang GJ, Swanson JM, Telang F: Dopamine in drug abuse and addiction: results of imaging studies and treatment implications. Arch Neurol 2007, 64:1575-1579.

84. Ma Q: Aryl hydrocarbon receptor degradation-promoting factor (ADPF) and the control of the xenobiotic response. Mol Interv 2007, 7:133-137.

85. Mayfield RD, Lewohl JM, Dodd PR, Herlihy A, Liu J, Harris RA: Patterns of gene expression are altered in the frontal and motor cortices of human alcoholics. J Neurochem 2002, 81:802-813.

86. Treadwell JA, Singh SM: Microarray analysis of mouse brain gene expression following acute ethanol treatment. Neurochem Res 2004, 29:357-369.

87. Thibault C, Lai C, Wilke N, Duong B, Olive MF, Rahman S, Dong H, Hodge CW, Lockhart DJ, Miles MF: Expression profiling of neural cells reveals specific patterns of ethanol-responsive gene expression. Mol Pharmacol 2000, 58:1593-1600.

88. Jones S, Bonci A: Synaptic plasticity and drug addiction. Curr Opin Pharmacol 2005, 5:20-25.

89. Sugiyama S, Di Nardo AA, Aizawa S, Matsuo I, Volovitch M, Prochiantz A, Hensch TK: Experience-dependent transfer of Otx2 homeoprotein into the visual cortex activates postnatal plasticity. Cell 2008, 134:508-520.

90. Stolt CC, Lommes P, Sock E, Chaboissier MC, Schedl A, Wegner M: The Sox 9 transcription factor determines glial fate choice in the developing spinal cord. Genes Dev 2003, 17:1677-1689.

91. Gohlke JM, Armant O, Parham FM, Smith MV, Zimmer C, Castro DS, 
Nguyen L, Parker JS, Gradwohl G, Portier CJ, Guillemot F: Characterization of the proneural gene regulatory network during mouse telencephalon development. BMC Biol 2008, 6:15.

92. Akahoshi E, Yoshimura S, Ishihara-Sugano M: Over-expression of AhR (aryl hydrocarbon receptor) induces neural differentiation of Neuro2a cells: neurotoxicology study. Environ Health 2006, 5:24.

93. Hanashima C, Li SC, Shen L, Lai E, Fishell G: Foxg I suppresses early cortical cell fate. Science 2004, 303:56-59.

94. Ohtsuka T, Ishibashi M, Gradwohl G, Nakanishi S, Guillemot F, Kageyama R: Hes I and Hes5 as notch effectors in mammalian neuronal differentiation. EMBO J 1999, | 8:2196-2207.

95. Ohtsuka T, Sakamoto M, Guillemot F, Kageyama R: Roles of the basic helix-loop-helix genes HesI and Hes 5 in expansion of neural stem cells of the developing brain. J Biol Chem 200I, 276:30467-30474.

96. Hatakeyama J, Bessho Y, Katoh K, Ookawara S, Fujioka M, Guillemot $F$, Kageyama R: Hes genes regulate size, shape and histogenesis of the nervous system by control of the timing of neural stem cell differentiation. Development 2004, 131:5539-5550.

97. Adolf B, Chapouton P, Lam CS, Topp S, Tannhauser B, Strähle U, Götz M, Bally-Cuif $L$ : Conserved and acquired features of adult neurogenesis in the zebrafish telencephalon. Dev Biol 2006, 295:278-293.

98. Grandel H, Kaslin J, Ganz J, Wenzel I, Brand M: Neural stem cells and neurogenesis in the adult zebrafish brain: origin, proliferation dynamics, migration and cell fate. Dev Biol 2006, 295:263-277.

99. Pellegrini E, Mouriec K, Anglade I, Menuet A, Le Page Y, Gueguen MM, Marmignon MH, Brion F, Pakdel F, Kah O: Identification of aromatase-positive radial glial cells as progenitor cells in the ventricular layer of the forebrain in zebrafish. J Comp Neurol 2007, 501:150-167.

100. Zupanc GK, Hinsch K, Gage FH: Proliferation, migration, neuronal differentiation, and long-term survival of new cells in the adult zebrafish brain. J Comp Neurol 2005, 488:290-3I 9.

I0I. Chapouton P, Jagasia R, Bally-Cuif L: Adult neurogenesis in nonmammalian vertebrates. Bioessays 2007, 29:745-757.

102. Broglio C, Gomez A, Duran E, Ocana FM, Jimenez-Moya F, Rodriguez F, Salas C: Hallmarks of a common forebrain vertebrate plan: specialized pallial areas for spatial, temporal and emotional memory in actinopterygian fish. Brain Res Bull 2005, 66:277-28I.

103. Salas C, Broglio C, Duran E, Gomez A, Ocana FM, Jimenez-Moya F, Rodriguez F: Neuropsychology of learning and memory in teleost fish. Zebrafish 2006, 3:157-171.

104. Dominguez-Escriba L, Hernandez-Rabaza V, Soriano-Navarro M, Barcia JA, Romero FJ, Garcia-Verdugo JM, Canales J]: Chronic cocaine exposure impairs progenitor proliferation but spares survival and maturation of neural precursors in adult rat dentate gyrus. Eur J Neurosci 2006, 24:586-594.

105. Eisch AJ, Harburg GC: Opiates, psychostimulants, and adult hippocampal neurogenesis: Insights for addiction and stem cell biology. Hippocampus 2006, 1 6:27|-286.

106. Venkatesan A, Nath A, Ming GL, Song H: Adult hippocampal neurogenesis: regulation by HIV and drugs of abuse. Cell Mol Life Sci 2007, 64:2120-2132.

107. Noonan MA, Choi KH, Self DW, Eisch AJ: Withdrawal from cocaine self-administration normalizes deficits in proliferation and enhances maturity of adult-generated hippocampal neurons. J Neurosci 2008, 28:2516-2526.

108. Kimmel CB, Ballard WW, Kimmel SR, Ullmann B, Schilling TF: Stages of embryonic development of the zebrafish. Dev Dyn 1995, 203:253-310.

109. Agilent Technologies [https://earray.chem.agilent.com/earray/]

II0. Stockhammer OW, Zakrzewska A, Hegedus Z, Spaink HP, Meijer $\mathrm{AH}$ : Transcriptome profiling and functional analyses of the zebrafish embryonic innate immune response to Salmonella infection. J Immunol 2009, 182:564I-5653.

III. R [http://www.r-project.org]

I 12. von Mering C, Jensen LJ, Kuhn M, Chaffron S, Doerks T, Kruger B, Snel B, Bork P: STRING 7 - recent developments in the integration and prediction of protein interactions. Nucleic Acids Res 2007:D358-362.

1 13. Tatusov RL, Fedorova ND, Jackson JD, Jacobs AR, Kiryutin B, Koonin EV, Krylov DM, Mazumder R, Mekhedov SL, Nikolskaya AN, Rao BS, Smirnov S, Sverdlov AV, Vasudevan S, Wolf YI, Yin JJ, Natale DA: The COG database: an updated version includes eukaryotes. BMC Bioinformatics 2003, 4:4I.
II4. zfishdb [http://mips.helmholtz-muenchen.de/zfishdb/]

II5. Pfaffl MW, Horgan GW, Dempfle L: Relative expression software tool (REST) for group-wise comparison and statistical analysis of relative expression results in real-time PCR. Nucleic Acids Res 2002, 30:e36.

116. Leucht C, Stigloher C, Wizenmann A, Klafke R, Folchert A, Bally-Cuif L: MicroRNA-9 directs late organizer activity of the midbrain-hindbrain boundary. Nat Neurosci 2008, I I:64 I-648.

117. Hauptmann G, Gerster T: Two-color whole-mount in situ hybridization to vertebrate and Drosophila embryos. Trends Genet 1994, 10:266.

1 18. Biomart [http://www.ensembl.org/biomart]

119. Clone/Gen ID converter software [http://idconverter.bio info.cnio.es/]

120. Homologene [http://www.ncbi.nlm.nih.gov/homologene]

12I. Li CY, Mao X, Wei L: Genes and (common) pathways underlying drug addiction. PLoS Comput Biol 2008, 4:e2. 